\title{
DISCONNECTION OF A BASAL GANGLIA CIRCUIT IN JUVENILE SONGBIRDS ATTENUATES THE SPECTRAL DIFFERENTIATION OF SONG SYLLABLES
}

\author{
Kevin C. Elliott ${ }^{3}$, Wei Wu ${ }^{1}$, Richard Bertram ${ }^{2}$, and Frank Johnson ${ }^{3,}{ }^{*}$ \\ ${ }^{1}$ Program in Neuroscience and Department of Statistics, Florida State University, Tallahassee, \\ Florida 32306-4301 \\ 2Program in Neuroscience and Department of Mathematics, Florida State University, \\ Tallahassee, Florida 32306-4301 \\ ${ }^{3}$ Program in Neuroscience and Department of Psychology, Florida State University, Tallahassee, \\ Florida 32306-4301
}

\begin{abstract}
Similar to language acquisition by human infants, juvenile male zebra finches (Taeniopygia guttata) imitate an adult (tutor) song by transitioning from repetitive production of one or two undifferentiated protosyllables to the sequential production of a larger and spectrally heterogeneous set of syllables. The primary motor region that controls learned song is driven by a confluence of input from two pre-motor pathways: a posterior pathway that encodes the adult song syllables and an anterior pathway that includes a basal ganglia-thalamo-cortical circuit. Like mammalian motor-learning systems, the songbird basal ganglia (BG) circuit is thought to be necessary for shaping juvenile vocal behavior (undifferentiated protosyllables) towards specific targets (the tutor's song syllables). Here, we tested the hypothesis that anterior pathway activity contributes to the process of protosyllable differentiation. Bilateral ablation of LMAN (lateral magnocellular nucleus of the anterior nidopallium) was used to disconnect BG circuitry at ages prior to protosyllable production and differentiation. Comparison to surgical controls revealed that protosyllables fail to differentiate in birds that received juvenile LMAN ablation - the adult songs of birds with $>80 \%$ bilateral LMAN ablation consisted of only one or two syllables produced with the repetitive form and spectral structure that characterizes undifferentiated protosyllables in normal juveniles. Our findings support a role for BG circuitry in shaping juvenile vocal behavior towards the acoustic structure of the tutor song and suggest that posterior pathway function remains in an immature 'default' state when developmental interaction with the anterior pathway is reduced or eliminated.
\end{abstract}

\section{Keywords}

vocal imitation; tutor; cortical development; ablation; undifferentiated

"Corresponding Author: Frank Johnson, Program in Neuroscience and Department of Psychology, Florida State University, Tallahassee, Florida 32306-4301, johnson@ psy.fsu.edu. 


\section{INTRODUCTION}

Despite different phylogenetic histories, vocal imitation is a dominant feature of the neurobehavioral development of songbirds and humans. In the zebra finch, vocal imitation is driven by dual pre-motor pathways located primarily in the anterior and posterior cerebrum (see Fig. 1). The anterior pathway - which includes a basal ganglia-thalamo-cortical circuit - generates subsong, the initial unstructured and low-amplitude babbling of juvenile birds (Aronov et al., 2008; Goldberg \& Fee, 2012). The posterior pathway - embedded within avian pallial (cortical) regions - interacts with the anterior pathway to drive vocalizations during plastic song and increases in neuronal, synaptic, and behavioral influence over the course of vocal development (Nordeen \& Nordeen, 1988b; Herrmann \& Arnold, 1991; Aronov et al. 2008). Behaviorally, the transition from subsong to plastic song is associated with the repetitive production of low-amplitude, spectrally undifferentiated song syllables known as protosyllables - that gradually differentiate into the spectrally heterogeneous structure of adult song syllables (Tchernichovski et al., 2001; Saar \& Mitra, 2008; Aronov et al., 2008; 2011). Production of the crystallized adult song pattern is directed by the posterior pathway (Long \& Fee, 2008).

Previous work established that anterior and posterior pathways must interact for vocal imitation to occur - ablation or inactivation of LMAN (the output nucleus of the anterior pathway) produces a rapid reduction in the variable structure of juvenile singing (Bottjer et al., 1984; Scharff \& Nottebohm, 1991; Olveczky et al., 2005; Aronov et al. 2008). In earlier studies where birds received juvenile LMAN ablation (35-50 days post-hatch, dph) the subsequent juvenile and adult songs often consisted of a small number of reduced-amplitude syllables, lacking in spectral differentiation and produced in a repetitive fashion (Bottjer et al., 1984; Scharff \& Nottebohm, 1991). These findings led to the view that juvenile LMAN ablation induces a premature crystallization of song (Scharff \& Nottebohm, 1991).

However, this work was conducted prior to the discovery of the role played by protosyllable production and differentiation in the process of vocal imitation (Tchernichovski et al. 2001) and similarity between the adult songs of birds that received juvenile LMAN ablation and normal juvenile protosyllables could not be discerned.

Here, we revisited the effects of juvenile LMAN ablation in order to test the hypothesis that anterior pathway activity contributes to the process of protosyllable differentiation. This hypothesis predicts that LMAN ablation prior to protosyllable production or differentiation will attenuate normal developmental increases in song length, song amplitude, and in the complexity of syllable spectral structure. The net effect of attenuating protosyllable differentiation should be adult song syllables that resemble protosyllables produced during early stages of plastic song. Thus, whereas the earlier view of premature crystallization suggests that juvenile LMAN ablation accelerates posterior pathway development (Scharff \& Nottebohm, 1991), the hypothesis tested here suggests that juvenile LMAN ablation will leave the posterior pathway in an immature state.

Bilateral LMAN ablations were carried out in two groups of juvenile birds: at $28 \mathrm{dph}$, prior to the production of protosyllables, and at $40 \mathrm{dph}$, prior to differentiation of protosyllables. To quantify song development relative to a known target, we ablated LMAN bilaterally in 
juvenile zebra finches that were raised in the presence of a single target of imitation (the song of one adult male zebra finch). Compared to surgical controls we found a significant relationship between the completeness of LMAN ablation (at either 28 or 40dph) and impairment of tutor imitation. This relationship was mediated by attenuation of normal developmental increases in song length, song amplitude and the complexity of syllable spectral structure. Moreover, the adult songs of birds with $>80 \%$ bilateral LMAN ablation consisted of one or two syllables that were produced with the repetitive form and acoustic structure that characterizes undifferentiated protosyllables in normal juvenile birds. Our findings provide strong support for the hypothesis that anterior pathway activity contributes to the process of protosyllable differentiation. The findings suggest that posterior pathway function remains in an immature 'default' state when developmental interaction with the anterior pathway is reduced or eliminated.

\section{MATERIALS AND METHODS}

\section{Animals and Environment}

Four pairs of adult male and female zebra finches from our breeding colony were each housed in cages $(38.00 \times 34.50 \times 34.50 \mathrm{~cm})$ placed in acoustic isolation chambers $(75.69 \times$ $40.39 \times 47.24 \mathrm{~cm}$ ). Each male and female pair was mated to produce the male offspring used for this study. Acoustic isolation chambers ensured that offspring were exposed exclusively to the song of one tutor, their father. A computer maintained both the photoperiod (14 hr. light, $10 \mathrm{hr}$. dark) and ambient temperature (set to $26^{\circ} \mathrm{C}$ ) for each chamber. Finches were provisioned daily with primarily millet-based assorted seed (400 Finch Blends, Jones Seed Company), a dietary supplement (Lafeber Finch Granules, Lafeber Company), water, cuttlebone, and grit. Nests with offspring were given more food as needed. All daily care procedures and experimental manipulations of the finches were reviewed and approved by the Florida State University Animal Care and Use Committee.

\section{Experimental Groups and General Procedure}

Our experimental design (Fig. 2) placed male offspring in one of three groups. The first group received electrolytic LMAN ablations at 28dph (28dph LMANX, N=11), near the onset of subsong production and well before protosyllable production. The second group received electrolytic LMAN ablations at 40dph (40dph LMANX, N=8), near the onset of plastic song and the emergence of protosyllables but before protosyllables begin to differentiate (this was confirmed by the behavior of surgical control birds included in this experiment). The third group consisted of sham surgical controls (CTL, N=9). All birds used in this experiment were the offspring of $\mathrm{N}=4$ adult male tutors: Tutor 109 ( 2 birds), Tutor 209 (4 birds), Tutor 309 (15 birds), and Tutor 409 (7 birds). The final group sizes were smaller as some ablation surgeries produced insufficient or bilaterally-asymmetrical LMAN ablations (see Statistics, below).

All birds were housed with their parents and clutch-mates until $40 \mathrm{dph}$ - this ensured that all birds received sufficient developmental exposure to their father's song, but this small-group setting (breeding pair plus 2-6 offspring) prevented us from obtaining identified preoperative recordings from individual birds. After $40 \mathrm{dph}$ all male offspring were housed 
singly in cages $(28.50 \times 21.00 \times 38.00 \mathrm{~cm})$ situated within sound recording chambers that provided complete visual and partial auditory isolation until $120 \mathrm{dph}$. Each recording chamber included an overhead (ceiling) microphone to monitor song development from 40 to $120 \mathrm{dph}$ (adulthood). The overhead location, while suitable for the capture of the spectral structure of singing, is not ideal for accurate measurement of sound amplitude - birds will typically produce song at cage positions that will result in sound contacting an overhead microphone off-axis. Therefore, song amplitude was assessed using a separate recording setup configured for accurate measurement of sound amplitude (described below). Birds that received either LMAN ablation or a control procedure at $28 \mathrm{dph}$ were returned to their parents and clutch-mates until $40 \mathrm{dph}$. Birds that received LMAN ablation or a control procedure at $40 \mathrm{dph}$ were housed singly in sound recording chambers immediately following surgery. Previous work indicates that exposure to a tutor until $40 \mathrm{dph}$ is sufficient for successful imitation of tutor song (Böhner 1990; Roper \& Zann 2006), a finding replicated here in the surgical control birds.

Once each male offspring reached $120 \mathrm{dph}$, they were transferred and housed singly in an acoustic isolation chamber (providing complete visual and auditory isolation) dedicated to the capture of their final adult songs. The cage, microphone, and microphone gain were set at fixed positions in this chamber for the duration of the experiment. The microphone was positioned beside the cage (facing the perch) to facilitate on-axis capture of song by the microphone, which is necessary for accurate measurement of sound amplitude. All birds (including tutors) were recorded in this chamber for a minimum of three days. Accurate measurement of amplitude and spectral structure differences between tutors and offspring were facilitated by using the same recording environment for capturing the songs of tutors and their adult offspring. Comparisons between the songs of tutors and their adult offspring were not made in earlier studies of the effects of juvenile LMAN ablation (Bottjer et al., 1984; Scharff \& Nottebohm, 1991).

\section{Surgery}

LMAN Ablation-We used a similar surgical methodology as Thompson et al. (2011). Finches were deeply anesthetized with Equithesin $(0.045 \mathrm{cc})$ and secured into a stereotaxic instrument. The skull was exposed by centrally incising the scalp and retracting the skin with curved forceps. The stereotaxic zero point was set at the bifurcation of the mid-sagittal sinus. Phosphate buffered saline (PBS, 0.02 molar) was used to clean and hydrate the area. Craniotomies were placed over the bilateral locations of LMAN, and ablation sites were located $1.3 \mathrm{~mm}$ and $1.8 \mathrm{~mm}$ laterally from the mid-sagittal line. The central 'core' region of LMAN was targeted with tungsten electrode wire (A-M Systems Inc.) lowered $2.8 \mathrm{~mm}$ from the surface of the brain, followed by passing $100 \mu \mathrm{A}$ current for 3 minutes. Following the surgery, the incision was treated with antiseptic, sealed with veterinary adhesive, and the offspring returned to their tutor's cage until $40 \mathrm{dph}$ (28dph LMANX), or housed singly as described above (40dph LMANX).

LMAN control surgery-Finches were deeply anesthetized with Equithesin $(0.045 \mathrm{cc})$ and then secured in a stereotaxic instrument. Craniotomies were placed around the approximate location of both LMAN. Following the surgery, the incision was treated with 
an antiseptic, sealed with veterinary adhesive, and the offspring returned to their cage. The timing of this control procedure (at 28- or 40-dph) did not alter behavioral or brain morphological outcomes and all control birds were therefore combined into a single control group.

\section{Morphological Analysis}

Volumetric Measurement and Neuron Counting-Following their final recordings, all adult offspring were overdosed with Equithesin $(0.1 \mathrm{cc})$ and perfused intracardially with saline followed by ice-cold $4 \%$ paraformaldehyde. Brains were post fixed overnight in $4 \%$ paraformaldehyde, and transferred to PBS until sectioning. Brains were separated into rostral and caudal halves through coronal blocking at a point separating HVC/RA and Area $\mathrm{X} / \mathrm{LMAN}$. The rostral tip of the zebra finch midbrain was used as a fiduciary landmark for the block. Each half was sectioned at $40 \mu \mathrm{m}$ along the coronal plane using a vibratome (Leica VT1000S). Using a section evaluation interval of 4 (i.e., every $4^{\text {th }}$ section was selected) reference sections were stained with thionin (a Nissl stain) and mounted onto microscope slides. We used an alternate set of thionin-stained sections for stereological neuron counting, also selected at a section evaluation interval of 4 .

The volumes of left and right LMAN, HVC, Area X, and RA were measured in the reference sections by tracing the Nissl-defined borders of each nucleus for every mounted section using a transmitted-light microscope (Leica DM 5000B) interfaced (by camera and automated stage) with a PC running Neurolucida 7.0 software (MicroBrightField, Williston, VT). Each nucleus was defined by distinctive differences in neuron size, density, and staining intensity from surrounding brain tissue. Tracings of LMAN borders were used to evaluate the completeness of bilateral ablation, whereas tracings of HVC, Area X, and RA were made to determine if LMAN ablation altered the volumetric development of these regions.

LMAN consists of a central intensely-stained magnocellular core surrounded by a parvicellular shell (Johnson \& Bottjer 1992). Although both core and shell receive input from the thalamus (DLM), only the LMAN core projects to RA (Johnson et al. 1995) and influences the acoustic structure of juvenile song (Bottjer et al., 1984; Olveczky et al., 2005). The efferent target of the shell - a region of arcopallium that is adjacent to the lateral edge of RA (see Fig. 1) - does not influence the acoustic structure of juvenile song but may play a role in the normative reduction of variability in phonology and syntax that occurs as birds reach adulthood (Bottjer \& Altenau 2010). Here, volume tracings in both LMANintact and LMAN-ablated birds were calculated based on the Nissl-defined borders of the magnocellular LMAN core. In birds where the LMAN ablations were incomplete, the tracings were used to document the anatomical position of the remaining portions of LMAN.

To verify that our electrolytic ablations targeted only LMAN and did not damage MMAN (the medial magnocellular nucleus of the anterior nidopallium, an adjacent song region that projects to HVC) we compared the positions of ablation sites for LMANX birds to the position of retrogradely-labeled MMAN neurons in a separate set of juvenile birds. As MMAN is not readily identifiable in Nissl-stained tissue the retrograde fluorescent tracers DiI and DIO (Life technologies) were injected into HVC of $\mathrm{N}=2$ juvenile birds ( $40 \mathrm{dph}$ ), 
followed by seven days for retrograde dye transport into MMAN. Coronal sections from these birds were used to determine the position of the lateral border of MMAN (relative to the midline), which was then compared to the medial border of LMAN ablations in coronal sections from 28- and 40-dph LMANX birds (relative to the midline). No overlap was found between the two borders (the average distance between the two borders was $360 \mu \mathrm{m}$ ) indicating that MMAN was spared in all birds that received juvenile LMAN ablation.

We also estimated the number of neurons in HVC, Area X, and RA to determine if juvenile LMAN ablation altered the development of neuron number in these regions. Stereological neuron counting in left and right HVC, Area X, and RA used the same microscope and PC as above, running Stereo Investigator 7.0 software (MicroBrightField, Williston, VT).

Stereology was based on the method of Bonthius et al. (2004), where neurons were counted in wet sections (to prevent shrinkage in section thickness), providing many more z-axis focal planes for stereology. Wet sections were prepared by gentle agitation in thionin for 20 seconds, followed by differentiation in PBS for two minutes. Use of a Nissl stain for stereological counting of neurons has been demonstrated to be as sensitive and accurate as use of a neuron-specific antibody for neuron counting (Hou et al. 2012). Thionin-stained sections were wet-mounted onto glass slides and cover slipped with PBS.

Initially, the borders of each song region were traced at low magnification (5x objective). Then neuronal nuclei were counted using the optical fractionator technique at high magnification (100x oil objective) with differential interference contrast (DIC) optics. At high magnification, use of wet sections (which preserved full section height) along with DIC facilitated visual discrimination between neuronal and non-neuronal nuclei. Neuronal nuclei were identified by a fringe of stained cytoplasm and the presence of one or two spherical, basophilic nucleoli, whereas glial nuclei were identified by the presence of little or no cytoplasmic staining and a small, irregular shaped nucleus containing numerous darkly stained granular nucleoli.

The optical fractionator technique combines systematic random sampling of counting sites with a counting box, the upper and lower boundaries of which are inset from the upper and lower surfaces of the section. In the $40 \mu \mathrm{m}$-thick wet sections used for counting, these insets (guard zones) were $10 \mu \mathrm{m}$, meaning that all counting occurred within a $20 \mu \mathrm{m}$ optical section centered within each section. The $10 \mu \mathrm{m}$ guard zones ensured that no split cells were counted. For each song region, the frequency of sampling and the counting box size were determined by a priori analyses that produced stable and low Gundersen coefficient of error $(m=1)$ values within a range of $0.04-0.1$. Gundersen coefficient of error $(m=1)$ values are a measure of the uncertainty associated with population estimates (Gundersen et al. 1999). A preliminary analysis using $\mathrm{N}=528 \mathrm{dph}$ LMANX birds, $\mathrm{N}=240$ dph LMANX birds, and $\mathrm{N}=6$ CTL birds of neuron number in HVC and Area X showed no group differences in the distribution of values as a function of LMAN ablation - therefore only RA neuron number was estimated in all birds.

Left and right hemisphere values for volumetric (LMAN, HVC, RA, Area X) and neuron number (RA) measures were combined to generate a single bilateral value for each measure in each bird. For data analysis, the resulting values for 28- and 40-dph LMANX birds were 
then expressed as 'percent control' (i.e., percentage of the CTL mean for each of the measures).

\section{Song Recording}

Starting at $40 \mathrm{dph}$, twice-weekly recording sessions monitored vocal development using a PC running sound-event triggered software (Avisoft Recorder, Avisoft Bioacoustics, Berlin, Germany) that sampled sound at a rate of $44 \mathrm{kHz}$. Each captured sound event was saved to the computer's hard drive as a time-stamped .wav file. To insure that all song bouts were recorded each day, capture settings on Avisoft Recorder were set to favor false positives (e.g., a series of calls or repeated pecking on the food dish). To eliminate files that did not contain song bouts we followed methods described in Wu et al. (2008). All .wav files were converted to .jpg images of the frequency ranges present in each file using the batch function in Spectrogram 12.0 (Visualization Software, Richard Horne). These images were then sorted using an image-pattern matching program (iMatch, Mario Westphal) to group .wav files that did not contain singing. All .wav files that did not contain singing were then batch deleted using a MATLAB script (Mathworks Inc., Natick, MA; Wu et al., 2008).

The acoustic isolation chambers that housed male and female pairs and their offspring (until the offspring were $40 \mathrm{dph}$ ) were also monitored for subsong. Although we could not distinguish between the vocalizations of individual juveniles within each chamber, the purpose of these recordings was to validate the onset of subsong. We noted that the onset of subsong typically began around $30 \mathrm{dph}$, a result observed in earlier literature (Arnold, 1975).

\section{Adult Song Analysis}

Percent Similarity to Tutor-Using adult songs from each bird (and their respective tutors) that were all recorded in the same acoustic isolation chamber, Sound Analysis Pro (SA+) v1.04 software (Tchernichovski et al., 2000) was used to generate a percent similarity score between each bird and their respective tutor (asymmetric comparison, a higher percentage represents more accurate song acquisition). For each bird, 50 song files were selected randomly from their final post-120dph recordings. All birds' song bouts were compared to a representative tutor song motif and the resulting percent similarity scores were averaged to generate a single score. Asymmetric percent similarity measures calculate spectral similarity between tutor and pupil songs and ignore spectral dissimilarity. This is appropriate for tutor-pupil comparisons as the process of song imitation results in facsimiles rather than duplications. Because asymmetric percent similarity measures ignore spectral dissimilarity the songs of two unrelated adult male zebra finches will produce 30-50\% similarity due to the species-typical frequency structure of zebra finch songs. As a result, the $y$-axis origin in our graph of the percent similarity data starts at 50 percent.

Motif Duration-Using the same 50 song files used to generate percent similarity, the adult motif of each bird (tutors as well as offspring) was measured to the nearest millisecond, and the motif durations of offspring were calculated as a percent of their respective tutor. The motif for each bird was defined as a single instance of the canonical syllable pattern that is repeated in whole or in part during each song bout. 
Song and Call Amplitude-Using the same 50 song files used to generate percent similarity, song amplitude values (in $\mathrm{dB}$ ) were calculated for each bird by taking the mean amplitude for each song syllable and averaging them into a single value. Average tutor song amplitude was also calculated by using 50 song files recorded from the same acoustic isolation chamber. To compare song amplitude values of adult offspring to their respective tutors, these values were also calculated as a percent tutor. The amplitude of distance calls was also analyzed using forty-five representative distance calls (Zann, 1985) taken from song files of each bird and their respective tutors. For each bird, call amplitudes were averaged to generate a single value.

\section{Analysis of Song Development}

Protosyllable Identification-Recordings from each CTL bird were used to identify the onset of protosyllable production. The transition from the repetitive production of protosyllables to the serial delivery of several different syllable types can be detected by measuring the period (elapsed time) between repeated, similar sounds (Tchernichovski et al. 2001). Using default settings in SA+, the periods between similar syllables were calculated from the song bouts of CTL birds at bi-weekly intervals across vocal development. A period of less than $150 \mathrm{msec}$ was used to identify song bouts that included the repetitive production of protosyllables.

Development of Syllable Spectral Variance-Song was measured at six different days selected from six consecutive weeks of development (40-46 dph, 54-60 dph, 68-74 dph, 82-88 dph, 96-102 dph, and 110-116 dph). For each day measured, the first 50 song files were selected for analysis. Individual syllables within the song files were segmented by amplitude using syllable threshold controls in Sound Analysis Pro (SA+) v1.04 software (Tchernichovski et al., 2000). To improve syllable segmentation, all song files were processed with a $600 \mathrm{~Hz}$ high-pass filter and an amplitude decrement of -10dB prior to analysis in SA+. Three measures of variance in syllable structure were analyzed across development: pitch (variation in frequency structure across each syllable), pitch goodness (variation in harmonic structure across each syllable), and entropy (variation in noise structure across each syllable). These variances (expressed as SD) were calculated for each syllable by SA+, using the default zebra finch settings (FFT window $=9.27 \mathrm{msec}$, window advance $=1.36 \mathrm{msec}$ ). We selected these syllable variance measures based on the findings of Tchernichovski et al. (2001), where a developmental increase in syllable complexity (measured as an increase in spectral variance across individual syllables) was observed during song learning. That is, whereas syllables produced during subsong are characterized by relatively little modulation of pitch, pitch goodness, or entropy, the highly structured syllables of adults are often produced by systematic, relatively wide modulation of these same features.

Six data files were generated for each bird - each data file separated by two weeks of development - and were normalized to the data file with the lowest syllable number. Variance measures for pitch, pitch goodness, and entropy were calculated for each of the six data files. The resulting average SD values for pitch, pitch goodness, and entropy were then normalized to the first data file ( $40-46 \mathrm{dph}$ ), followed by an averaging of all three 
normalized variance measures for each of the six time points. This procedure produced a single composite measure of syllable variance normalized to the first recording week, meaning that increasingly positive values across development reflect an increase in syllable complexity (as observed by Tchernichovski et al. 2001). Finally, in order to make group comparisons, difference scores for each bird were calculated by subtracting the normalized variance measures for the first week (40-46 dph) from the last week (110-116 dph).

\section{Statistics}

All between- or within-group difference measures were analyzed using ANOVAs and appropriate post-hoc tests (GraphPad Prism 5, GraphPad Software, Inc.): One-way ANOVA with Tukey post-hoc comparisons were used for measures with one independent variable and two-way ANOVA with Bonferroni post-hoc comparisons were used for measures with two independent variables. Data from a subset of birds were omitted from this analysis due to incomplete LMAN ablation ( $\mathrm{N}=3$ birds with $>50 \%$ of LMAN remaining) and interhemispheric asymmetry in LMAN ablation ( $\mathrm{N}=2$ birds with greater than $25 \%$ asymmetry in the volume of LMAN remaining between left and right hemispheres). As a result, sample sizes for statistical analyses were: 28dph LMANX (N=7), 40dph LMANX (N=7), CTL $(\mathrm{N}=9)$.

\section{RESULTS}

\section{Confirmation of LMAN ablation}

Figure 3 depicts schematic representations of three bilateral coronal sections containing rostral, central, and caudal LMAN for both 28- and 40-dph LMANX groups. LMAN in each section shows the total amount of LMAN ablated for each group, color-scaled according to number of birds with partial or complete damage to LMAN in each section. These schematic representations show the spatial extent of LMAN ablations and reveal that LMAN ablation in both 28- and 40-dph LMANX groups occurred most consistently within central and caudal regions of LMAN (i.e., birds with incomplete ablation were more likely to show sparing of rostral LMAN).

Quantification of LMAN ablation is shown in Figure 4, where column-scatterplots depict remaining bilateral LMAN volumes for birds in each group, expressed as a percentage of the mean intact bilateral LMAN volume in the CTL group. Each data point represents an individual bird, gray-scaled according to the completeness of the LMAN ablation (i.e., darker circles indicate greater LMAN ablation). Both the 28- and 40-dph LMANX groups had significantly smaller LMAN volumes than the CTL group $\left(\mathrm{F}_{(2,22)}=82.76, \mathrm{p}<0.01\right)$. Pairwise comparisons reveal that both 28- and 40-dph LMANX birds had smaller LMAN volumes than CTL birds ( $\mathrm{p}<0.01$ for both comparisons), but they were not different from each other. 28- and 40-dph LMANX groups showed statistical equivalence in the extent of LMAN ablation: values ranged from $0 \%$ to $31 \%$ LMAN remaining in the 28dph LMANX group and $0 \%$ to $27 \%$ LMAN remaining in the 40dph LMANX group. 


\section{The development of HVC and Area X volumes is not influenced by LMAN ablation at 28- or 40-dph}

Prior work by Bottjer et al. (1985) and Nordeen \& Nordeen (1988a,b) established an increase in HVC volume and neuron number during the sensitive period of zebra finch song development. To test whether LMAN ablation at 28- or 40-dph affected HVC development, adult $\mathrm{HVC}$ volumes were calculated in all birds. Figure 5A shows that HVC volume did not differ significantly as a function of LMAN ablation $\left(\mathrm{F}_{(2,21)}=2.58, \mathrm{p}=0.10\right.$; HVC volume for one bird in the CTL group was not obtained due to damage during tissue processing). The nucleus Area X, which also increases in volume and neuron number during song development (Bottjer et al. 1985), and receives afferent input from LMAN (Vates \& Nottebohm, 1995), was likewise examined. Figure 5B shows that Area $X$ volume also did not differ significantly as a function of LMAN ablation $\left(\mathrm{F}_{(2,22)}=1.55, \mathrm{p}=0.24\right)$. Thus, developmental mechanisms that establish the adult volumes of HVC and Area X appear to function normally following significant reduction in the size of LMAN. The normal volumetric development of $\mathrm{HVC}$ is of particular interest as this region encodes the adult song pattern (Long et al. 2010), and individual variation in HVC volume correlates with several measures of song complexity (Airey \& DeVoogd, 2000). Therefore, differences in vocal imitation between CTL birds and birds with juvenile LMAN ablation cannot be due to a gross anatomical deficit in HVC.

\section{LMAN ablation at 28- and 40-dph alters the morphological development of RA}

Earlier work by Bottjer et al. (1986) demonstrated that RA shows a volumetric increase during zebra finch song development that is due to a decrease in neuronal density, resulting in a stable number of RA neurons across development. Nevertheless, afferent terminals from LMAN are necessary for the survival of a subpopulation of RA neurons during a specific period of development - LMAN ablation at 20dph results in the apoptotic death of approximately 50\% of RA neurons (Johnson \& Bottjer, 1994; Akutagawa \& Konishi, 1994; Johnson et al., 1997), whereas LMAN ablation at 40dph (or in adult birds) no longer produces detectable neuron death in RA (Johnson \& Bottjer, 1994; Akutagawa \& Konishi, 1994). Therefore, we examined whether LMAN ablation at 28- or 40-dph affected the development of RA volume and neuron number.

Figure 6A shows that RA volume varied as a function of LMAN ablation $\left(\mathrm{F}_{(2,19)}=6.16, \mathrm{p}\right.$ $<0.01$; RA volume and neuron number data from two birds within the 28dph LMANX group and one from the CTL group were not obtained due to damage during tissue processing). Pairwise comparisons showed statistical significance only between the 28dph LMANX and CTL birds ( $\mathrm{p}<0.01$ ). Figure 6B shows that RA neuron number also varied as a function of LMAN ablation $\left(\mathrm{F}_{(2,19)}=5.57, \mathrm{p}=0.01\right)$. Pairwise comparisons revealed that RA neuron number in both 28- and 40-dph LMANX birds was significantly smaller than in CTL birds $(\mathrm{p}<0.05)$. However, Figure 6B also shows a downward compression (rather than a downward shift) in the distribution of data points for 28- and 40-dph LMANX groups, towards the lower end of the distribution of CTL values. Wide individual variation in HVC, Area X, and RA morphology is a normative feature of the zebra finch song system (see CTL group in Figs. 5 and 6, and Bottjer et al., 1985; Airey \& DeVoogd, 2000). Thus, the effect of LMAN ablation at 28- or 40-dph appears to be one of reducing individual variation in RA 
neuron number, resulting in values centered near the low end of the distribution observed in CTL birds. Therefore, it seems unlikely that the large differences in vocal imitation between CTL birds and both LMANX groups would be due solely to differences in RA volume or neuron number. Indeed, individual variation in CTL RA volume (values ranged from $0.12 \mathrm{~mm}^{3}$ to $0.26 \mathrm{~mm}^{3}$ per unilateral RA) and CTL RA neuron number (values ranged from 14,500 to 28,900 neurons per unilateral RA) did not correlate significantly with the ability to imitate a tutor - CTL birds with small RA volume and neuron number values were just as likely to accurately copy their tutor as CTL birds with large values for RA volume and neuron number (regression of percent similarity to tutor against $R A$ volume: $R^{2}=0.20, p=$ 0.31 ; against RA neuron number: $\mathrm{R}^{2}=0.07, \mathrm{p}=0.56$ ).

\section{LMAN ablation at 28- or 40-dph produces similar deficits in spectral similarity to the tutor song, and attenuates imitation of the duration and amplitude of the tutor song}

Figure 7 shows example frequency spectrograms of adult song bouts from birds within each group, along with a song bout from their tutor. Below the tutor's spectrogram are letters, each corresponding to a different syllable within the tutor's song motif. Tutor syllables copied by CTL sons are indicated below their respective spectrograms. Examination of frequency spectrograms from birds with juvenile LMAN ablation (at either 28- or 40-dph) reveals shortened motifs that contained fewer syllables, produced at reduced amplitude (amplitude is gray-scaled in the frequency spectrograms). In birds where a $>20 \%$ portion of LMAN remained (birds 309V and 309Y), some syllables resembled particular tutor syllables (indicated by transparent colors). In birds where $\_20 \%$ of LMAN remained (birds 3090 and $309 \mathrm{~W}$ ) the motif was reduced to one or two repeated syllables that could not be unambiguously identified as belonging to the tutor's motif. Of particular interest is the observation that birds with LMAN ablation near the onset of subsong production and before protosyllable production (28-dph LMANX) and birds with LMAN ablation near the onset of plastic song (when protosyllables first emerge) but before protosyllable differentiation (40dph LMANX) show a similar behavioral deficit. The common feature between the two groups is that LMAN ablation occurred prior to the differentiation of protosyllables - there appeared to be no cumulative behavioral benefit conferred by LMAN activity between 28 and $40 \mathrm{dph}$.

Quantification of spectral similarity-To quantify the visually-apparent reduction in the accuracy of tutor song imitation observed in both groups that received LMAN ablation (see Fig. 7), adult song recordings from each bird were compared against their respective tutor using the asymmetric percent similarity function in SA+ (all birds recorded in the same acoustic isolation chamber for this comparison - see Methods). Figure 8 shows that percent similarity measures varied as a function of LMAN ablation $\left(\mathrm{F}_{(2,22)}=11.86, \mathrm{p}<0.001\right)$. Pairwise comparisons revealed that both 28- and 40-dph LMANX birds were less similar to their respective tutor than CTL birds ( $\mathrm{p}<0.01$ for both comparisons), but they were not different from each other. Thus, LMAN ablation at either 28- or 40-dph produces a similar deficit in imitation of the spectral structure of tutor song. Moreover, this attenuation of tutor imitation was not associated with a lack of vocal practice (mean song bout production during development did not differ between groups, $\mathrm{F}_{(2,22)}<1$, data not shown). 
The distribution of data points in Figure 8 also shows that within the 28- and 40-dph LMANX groups, greater LMAN ablation is associated with reduced similarity to the tutor song. We quantified this relationship and found that percent similarity to the tutor song is correlated with the amount of LMAN remaining following ablation when data from both 28and 40-dph groups are combined $\left(\mathrm{R}^{2}=0.45, \mathrm{p}<0.01\right)$. This finding indicates that LMAN retains partial function even in a substantially reduced state.

Quantification of motif duration-Over the course of plastic song, zebra finches transition from repetitive production of undifferentiated protosyllables to the serial production of most or all of their tutor's syllables, resulting in a gradual lengthening of the motif as song is learned (Tchernichovski et al., 2001). To quantify the apparent difference in adult motif duration between CTL and LMANX birds (see Fig. 7), the duration (in msec) of each bird's adult motif was compared against their respective tutor. Figure 9 shows that motif duration varied as a function of LMAN ablation $\left(\mathrm{F}_{(2,22)}=12.15, \mathrm{p}<0.001\right)$. Pairwise comparisons revealed that both 28- and 40-dph LMANX birds had shorter motifs compared to their respective tutor than CTL birds ( $\mathrm{p}<0.01$ for both comparisons), but they were not different from each other. Furthermore, no bird in either the 28- or 40-dph LMANX groups had a motif that was greater than the duration of the tutor motif (i.e., motif durations for all ablation birds were reduced relative to the tutor). Thus, whereas CTL birds successfully imitated the motif duration of their tutor, LMAN ablation at either age (28- or 40-dph) attenuated this aspect of tutor imitation, resulting in shortened motifs composed of only one or a few syllables.

Quantification of motif amplitude-Song amplitude increases as zebra finches transition from subsong to plastic song to adult song (Aronov et al., 2008; Naie \& Hahnloser, 2011). To quantify the apparent difference in the amplitude of adult song between CTL and LMANX birds (see Fig. 7), the average amplitude of each bird's adult motif syllables was compared to that of their respective tutors. Figure 10A shows song amplitude varied as a function of LMAN ablation $\left(\mathrm{F}_{(2,22)}=6.81, \mathrm{p}<0.01\right)$. Pairwise comparisons revealed that the amplitudes of both 28- and 40-dph LMANX birds were less similar and reduced to their respective tutor than CTL birds ( $p<0.01$ and $<0.05$ for 28 - and 40dph LMANX, respectively), but they were not different from each other. Thus, whereas CTL birds successfully imitated the motif amplitude of their tutor, LMAN ablation at either age (28- or 40-dph) attenuated this aspect of tutor imitation.

Because little is presently known about the neural control of song amplitude, we wondered whether the amplitude reduction in both 28- and 40-dph LMANX birds was a general property of all vocal production, or specific to song. Therefore, the amplitude of distance calls captured during recorded sessions was also measured. Figure 10B shows call and song amplitude in decibels $(\mathrm{dB})$ for each bird. Results from a two-way ANOVA show that vocalization amplitude varied as a function of the type of vocalization produced $\left(\mathrm{F}_{(1,40)}=\right.$ $46.16, \mathrm{p}<0.01)$. That is, there was a difference in amplitude of song vs. call. General amplitude for each group (average of both song and call) varied as a function of LMAN ablation $\left(\mathrm{F}_{(2,40)}=7.19, \mathrm{p}<0.01\right)$. Interactions between type of vocalization and group measures show an effect: amplitude of vocalization varied as a function of the type of 
vocalization produced and as a function of LMAN ablation $\left(\mathrm{F}_{(2,40)}=3.66, \mathrm{p}<.05\right)$.

Pairwise comparisons revealed that both 28- and 40-dph LMANX birds had reduced song amplitude compared to CTL birds ( $\mathrm{p}<0.05$ for both comparisons), but they were not different from each other. However, call amplitude was not different between groups ( $\mathrm{t}$ value $<1.12$ for all comparisons, $\mathrm{p}>0.80$ for all comparisons). Mean differences between call and song amplitude for each group show greater song and call disparity in LMANX birds compared to CTL (CTL - 5.33dB, 28dph LMANX - 16.12dB, and 40dph LMANX $14.90 \mathrm{~dB})$. These data demonstrate that birds with LMAN ablation retain the vocal-motor capacity to produce high-amplitude sounds, so the amplitude attenuation that characterizes their singing cannot be attributed to general vocal-motor impairment.

\section{LMAN ablation at 28- or 40 -dph attenuates the differentiation of protosyllables}

Development of syllable spectral complexity-In order to understand how LMAN ablation influenced the differentiation of protosyllables, we analyzed singing across 6 weeks of vocal development in all birds (beginning at 40-46 dph). We were guided in this analysis by the earlier findings of Tchernichovski et al. (2001) where it was demonstrated that syllable complexity (measured as spectral variation across individual syllables) increases as juvenile protosyllables differentiate into spectrally heterogeneous adult syllables. Figure 11 shows a line-graph representing a composite value of syllable spectral variance across six weeks of vocal development. Values were normalized against the first week of singing for each bird in order to account for differences in the spectral structure of the tutor songs (each bird raised by one of four different tutors, see Methods).

Data for the CTL birds are a replication of the findings of Tchernichovski et al. (2001), where syllable spectral variance shows a significant increase over development $\left(\mathrm{F}_{(5,53)}=\right.$ 8.176, $\mathrm{p}<0.01$ ). In contrast, birds with LMAN ablation from both 28- and 40-dph LMANX groups failed to show any significant increase in syllable spectral variance during development (28-dph LMANX: $F_{(5,41)}=1.458$, 40-dph LMANX: $F_{(5,35)}=0.779$; data for one bird in the 40-dph LMANX group were lost due to computer malfunction). These within-group comparisons were confirmed by a between-group comparison of the slope of spectral variance values across vocal development $\left(\mathrm{F}_{(2,22)}=9.941\right)$ : 28 - and 40-dph birds were significantly different from CTL birds ( $28 \mathrm{dph}, \mathrm{p}<0.05 ; 40 \mathrm{dph}, \mathrm{p}<0.01)$, but not significantly different from each other.

\section{Acoustic similarity of juvenile protosyllables and the adult syllables of} LMANX birds-The flattened developmental trajectories of LMANX birds shown in Figure 11 suggest that LMAN ablation attenuates the differentiation of juvenile protosyllables. If so, one would expect that the acoustic properties of adult syllables from birds with LMAN ablation should resemble the acoustic properties of normal juvenile protosyllables. To test this expectation we selectively analyzed song bouts that contained protosyllables from the initial recordings of juvenile CTL birds. As juvenile birds transition from subsong to plastic song their singing contains a mix of subsong elements and protosyllables, and song bouts that contained mostly or all subsong elements were excluded from this analysis. Figure 12A shows frequency spectrograms of protosyllable production by CTL birds between $42-49 \mathrm{dph}$. Similarity to the adult songs of birds with complete LMAN 
ablation is evident in both the repetitive form and the low spectral and temporal complexity of individual syllables (compare Fig. 12A to 3090 and 309W in Fig. 7). To quantify this apparent similarity, we compared spectral and temporal variances of: 1.) juvenile protosyllables from CTL birds, 2.) adult syllables from LMANX birds with $20 \%$ LMAN remaining (LMANX birds with > 20\% LMAN remaining showed evidence of tutor imitation, see Fig. 7), and 3.) adult syllables from CTL birds. Syllable spectral variance was calculated as before (Fig. 11) whereas the coefficient of variation (CV) was used to measure the structure of syllable temporal variance.

Results from one-way ANOVAs indicated significant group differences for syllable spectral $\left(F_{(2,18)}=9.24, p<0.01\right.$, Fig. 12B $)$ and temporal $\left(F_{(2,18)}=11.41, p<0.001\right.$, Fig. 12C $)$ variances. Pairwise comparisons showed that juvenile CTL protosyllables and adult syllables from LMANX birds did not differ in spectral (Fig. 12B) or temporal (Fig. 12C) variance, suggesting an acoustic equivalence of syllables produced by the two groups. While we observed the expected increase in syllable spectral and temporal variance between juvenile CTL protosyllables and adult CTL syllables ( $\mathrm{p}<0.01$, both comparisons), we also observed the same pattern of significance between the adult syllables of LMANX birds and adult CTL syllables ( $\mathrm{p}<0.01$, both comparisons). Together, the data in Figures 11 and 12 show that LMAN ablation attenuates the differentiation of juvenile protosyllables and results in adult songs that share the repetitive form and acoustic structure of undifferentiated juvenile protosyllables.

\section{DISCUSSION}

Our goal in this experiment was to elucidate the role of a basal ganglia-thalamo-cortical circuit (the anterior pathway) in the process of songbird vocal imitation. We disconnected the anterior pathway in juvenile birds (via ablation of LMAN) in order to test the hypothesis that neural activity conveyed by the anterior pathway contributes to the differentiation of juvenile protosyllables. This hypothesis predicts that LMAN ablation prior to the production ( $28 \mathrm{dph}$ ) or differentiation ( $40 \mathrm{dph}$ ) of juvenile protosyllables should impair tutor imitation by attenuating developmental increases in motif length, motif amplitude, and syllable spectral complexity. A further prediction is that the net result of attenuating protosyllable differentiation should be adult songs produced with a similar repetitive form and acoustic structure as juvenile protosyllables. Experimental results confirmed the hypothesis - we found that LMAN ablation at either age impaired tutor imitation by attenuating developmental increases in motif length, motif amplitude, and syllable spectral complexity. Moreover, we found that the song syllables of adult birds with $>80 \%$ juvenile LMAN ablation were produced with the same repetitive form and acoustic structure as juvenile protosyllables. An implication of these findings is that the posterior (descending cortical) pathway of the song control network remains in a functionally immature state (i.e., it continues to generate undifferentiated protosyllables) when developmental interaction with the anterior pathway is eliminated.

Our findings also confirm and extend findings from classic studies of the effects of juvenile LMAN ablation on vocal development (Bottjer et al., 1984; Scharff \& Nottebohm, 1991). We confirmed that the adult songs of birds with juvenile LMAN ablation are abnormally 
short, repetitive and produced at low amplitude. Findings were extended by ablating LMAN at two specific ages - at $28 \mathrm{dph}$ (prior to the production of protosyllables) and at $40 \mathrm{dph}$ (prior to the differentiation of protosyllables) - and comparing the adult songs of these birds to their tutor's song. Both groups showed a similar impairment of tutor imitation (Fig. 8), indicating that LMAN activity between 28 and 40 dph does not result in cumulative progress towards tutor imitation. Interestingly, a cumulative influence of LMAN activity was noted by Bottjer et al. (1984) and by Scharff and Nottebohm (1991) in birds singing plastic song (see Fig. 2), where syllables were already partially differentiated and vocal patterning had begun to emerge. In these birds, LMAN ablation resulted in an immediate cessation of further syllable differentiation (cf. Olveczky et al., 2005), but the partially differentiated vocal patterns were retained into adulthood. In the context of the present findings, it appears that LMAN activity will only produce a cumulative effect on vocal output once the process of syllable differentiation is underway.

The classic findings of Bottjer et al. (1984) and Scharff and Nottebohm (1991) were also extended by our comparisons of song amplitude against the amplitude of distance calls (Fig. 10). These comparisons demonstrated that adult birds with juvenile LMAN ablation retain the vocal-motor capacity to produce high-amplitude sounds. Thus, the amplitude attenuation that characterizes their singing cannot be attributed to a general vocal-motor impairment - it is specific to the forebrain motor circuit that controls learned vocalizations. Finally, by measuring the development of syllable spectral structure we concluded that juvenile LMAN ablation attenuates the differentiation of protosyllables (Fig. 11). This interpretation differs from the prior assessment that juvenile LMAN ablation induces an early crystallization of song (Scharff \& Nottebohm, 1991). While the two interpretations may appear to be two sides of the same coin, early crystallization suggests that LMAN ablation hastens the close of vocal development, whereas attenuation of protosyllable differentiation suggests that LMAN ablation prevents the opening of vocal development. In support of the latter interpretation we demonstrated that juvenile LMAN ablation results in adult songs that share the repetitive form and acoustic structure of undifferentiated juvenile protosyllables (Fig. 12).

\section{Dual pre-motor interaction is required for vocal imitation}

An interesting feature of the forebrain network for song control is that posterior and anterior pathways can each generate normative pre-motor activity in the absence of the other. The posterior pathway continues to produce the learned vocal pattern following adult LMAN ablation (Bottjer et al., 1984; Kao \& Brainard, 2006; Thompson et al., 2011) and the anterior pathway continues to produce subsong following juvenile HVC ablation (Aronov et al., 2008; Thompson et al., 2011). However, our behavioral findings indicate that posterior and anterior pathways must begin to interact near the onset of plastic song in order for protosyllable differentiation (and vocal imitation) to occur. Our current understanding of the architecture of the song system suggests that convergent synaptic interaction between LMAN and HVC could occur at RA and/or Area X (see Fig. 1). While our behavioral data do not allow us to selectively attribute attenuation of song development to one or the other region, our morphological measurements (volume and neuron number) indicate that the development of Area $X$ was not affected by loss of LMAN input (see Fig. 5B and results). 
LMAN ablation could have altered the development of the synaptic architecture or physiology of Area X, which we did not assess here. We did find that the development of RA neuron number was affected by the loss of LMAN input at 28- or 40-dph (Fig. 6). RA neuron number values in these birds fell to the lower end of the distribution of values measured in CTL birds.

To a degree, interpretation of the effect of LMAN ablation on RA neuron number is complicated by the observation that CTL birds with a similar number of RA neurons (i.e., CTL birds with neuron number values in the lower end of the distribution) successfully imitated the tutor song. Nevertheless, juvenile LMAN ablation could have impaired tutor imitation by inducing neuronal atrophy in RA - degenerative changes in RA of developing female zebra finches are associated with the inability to produce learned adult song (Nordeen \& Nordeen, 1988a). However, the uniformly low RA neuron number in birds that received juvenile LMAN ablation could also indicate attenuated development in the number and/or pattern of synapses that RA neurons receive from extrinsic (HVC) or intrinsic sources (connections between RA interneurons and RA projection neurons). Therefore, another possibility is that LMAN ablation interferes with the developmental addition and patterning of intrinsic and extrinsic synapses in RA, which could account for some or all aspects of the attenuated vocal development that we observed. It could be that the LMAN input to RA is necessary to guide the portion of HVC input that arrives during subsong and plastic song (Konishi \& Akutagawa, 1985; Nordeen \& Nordeen, 1988b; Mooney 1992). Anterograde tract-tracing indicates that $\mathrm{HVC}$ axon terminals do grow into RA of birds with juvenile LMAN ablation (F. Johnson, unpublished data) but LMAN could still be necessary for the formation of specific patterns of HVC-RA synaptic connectivity. Notably, juvenile LMAN ablation is known to rapidly alter some aspects of HVC synaptic architecture and physiology in RA (Kittelberger \& Mooney, 1999). However, whether these changes to posterior pathway architecture persist into adulthood is currently unknown and warrants further study.

There are also indirect routes of interaction between LMAN and HVC that could play an important role in protosyllable differentiation and vocal imitation. LMAN activity can be indirectly conveyed to HVC via midbrain dopaminergic neurons that project to HVC (Hamaguchi \& Mooney, 2012) or indirectly from its connections to RA, which contains both direct and indirect connections to HVC (Roberts et al. 2008). The behavioral significance of this indirect signaling from LMAN to HVC has yet to be fully elucidated, but such signaling would presumably be attenuated following LMAN ablation. Although we found that the development of HVC morphology was not altered by juvenile LMAN ablation (Fig. 6A), the absence of a dopaminergic signal within HVC would more likely have effects on synaptic architecture and physiology (Tritsch \& Sabatini, 2012).

Interestingly, once the process of vocal imitation is complete the effects of LMAN ablation are limited to a reduction in the acoustic variability of individual syllables and inter-syllable durations (Kao \& Brainard, 2006; Thompson et al., 2011). Because we are the first to measure and report adult song amplitude as a function of juvenile LMAN ablation, we also measured the amplitude of pre- and post-operative songs in a group of adult birds that received bilateral LMAN ablation ( $\mathrm{N}=6$, these birds were originally prepared by Thompson et al., 2011). On average, the difference in the mean amplitude of pre- vs. post-operative 
songs was quite small $(0.70 \mathrm{~dB})$ relative to the $>10 \mathrm{~dB}$ differences observed between CTL birds and birds that received juvenile LMAN ablation (Fig. 10A,B). Therefore, as with vocal patterning, the anterior pathway plays little role in the maintenance of the amplitude of the learned adult song.

\section{Anterior pathway activity and the differentiation of protosyllables}

Although anterior pathway influence over vocal production declines over development, the variable acoustic topography of vocalizations produced by the anterior pathway persists into adulthood (Aronov et al., 2008; Thompson et al., 2011). Moreover, recent studies in adult birds show that this variable topography can be brought under operant (goal-directed) control to bias vocal output towards specific acoustic targets (Andalman \& Fee, 2009; Sober \& Brainard, 2009; Charlesworth et al., 2012). Interestingly, while LMAN is required for the initial expression of this bias, LMAN itself appears to play no role in the calculation of the bias (Charlesworth et al., 2012). LMAN is also not needed for the later expression of this bias, as operantly-driven changes to song are eventually encoded within the posterior pathway (Andalman \& Fee, 2009; Sober \& Brainard, 2009; Charlesworth et al., 2012).

If extended to juvenile birds entering the developmental phase of plastic song, the above findings suggest that modulation of anterior pathway activity may generate the pre-motor bias that drives a small set of pluripotent protosyllables to differentiate towards a variety of acoustically-rich targets of imitation (i.e., the individual syllables of the tutor song). Protosyllables are the first vocal sounds driven primarily by the posterior pathway (Aronov et al., 2008; 2011) and our results suggest that protosyllables remain in a largely undifferentiated state when LMAN ablation occurs prior to their production or differentiation. It follows that key aspects of the physiology of the posterior pathway remain in an immature 'default' state when developmental interaction with the anterior pathway is prevented. Future identification of these aspects of posterior pathway physiology may reveal how basal ganglia circuits shape the development of cortical pathways for learned behavior.

\section{Acknowledgments}

Author contributions: KCE conducted all experimental work and collection of morphological and behavioral data. All authors contributed to data analysis and manuscript preparation. The authors thank Dr. Ofer Tchernichovski for use of the SA+ software and Dr. Richard Hyson for helpful suggestions on the manuscript. This work was supported by the National Institutes of Health (DC02035) and the National Science Foundation (IOS-1146607).

\section{REFERENCES}

1. Airey DC, DeVoogd TJ. Greater song complexity is associated with augmented song system anatomy in zebra finches. Neuroreport. 2000; 11:2339-2344. [PubMed: 10923697]

2. Akutagawa E, Konishi M. Two separate areas of the brain differentially guide the development of a song control nucleus in the zebra finch. Proc Natl Acad Sci USA. 1994; 91:12413-12417. [PubMed: 7809051]

3. Andalman AS, Fee MS. A basal ganglia-forebrain circuit in the songbird biases motor output to avoid vocal errors. Proc Natl Acad Sci USA. 2009; 106:12518-12523. [PubMed: 19597157]

4. Arnold AP. The effects of castration on song development in zebra finches (Poephila guttata). J Exp Zool. 1975; 191:261-278. [PubMed: 1113072]

5. Aronov D, Andalman AS, Fee MS. A specialized forebrain circuit for vocal babbling in the juvenile songbird. Science. 2008; 320:630-634. [PubMed: 18451295]

Dev Neurobiol. Author manuscript; available in PMC 2015 June 01. 
6. Aronov D, Veit L, Goldberg JH, Fee MS. Two distinct modes of forebrain circuit dynamics underlie temporal patterning in the vocalizations of young songbirds. J Neurosci. 2011; 31:16353-16368. [PubMed: 22072687]

7. Böhner J. Early acquisition of song in the zebra finch, Taeniopygia guttata. Anim Behav. 1990; 39:369-374.

8. Bonthius DJ, McKim R, Koele L, Harb H, Karacay B, Mahoney J, Pantazis NJ. Use of frozen sections to determine neuronal number in the murine hippocampus and neocortex using the optical disector and optical fractionator. Brain Research Protocols. 2004; 14:45-57. [PubMed: 15519951]

9. Bottjer SW, Miesner EA, Arnold AP. Forebrain lesions disrupt development but not maintenance of song in passerine birds. Science. 1984; 224:901-903. [PubMed: 6719123]

10. Bottjer SW, Glaessner SL, Arnold AP. Ontogeny of brain nuclei controlling song learning and behavior in zebra finches. J Neurosci. 1985; 5:1556-1562. [PubMed: 4009245]

11. Bottjer SW, Miesner EA, Arnold AP. Changes in neuronal number, density and size account for increases in volume of song-control nuclei during song development in zebra finches. Neurosci Lett. 1986; 67:263-268. [PubMed: 3737014]

12. Bottjer SW, Altenau B. Parallel pathways for vocal learning in basal ganglia of songbirds. Nat Neurosci. 2010; 13:153-155. [PubMed: 20023650]

13. Charlesworth JD, Warren TL, Brainard MS. Covert skill learning in a cortical-basal ganglia circuit. Nature. 2012; 486:251-255. [PubMed: 22699618]

14. Goldberg JH, Fee MS. A cortical motor nucleus drives the basal ganglia-recipient thalamus in singing birds. Nat Neurosci. 2012; 15:620-627. [PubMed: 22327474]

15. Gundersen HJ, Jensen EB, Kiêu K, Nielsen J. The efficiency of systematic sampling in stereology--reconsidered. J Microsc. 1999; 193:199-211. [PubMed: 10348656]

16. Herrmann K, Arnold AP. The development of afferent projections to the robust archistriatal nucleus in male zebra finches: a quantitative electron microscopic study. J Neurosci. 1991; 11:2063-2074. [PubMed: 2066775]

17. Hamaguchi K, Mooney R. Recurrent interactions between the input and output of a songbird cortico-basal ganglia pathway are implicated in vocal sequence variability. J Neurosci. 2012; 32:11671-11687. [PubMed: 22915110]

18. Johnson F, Bottjer SW. Growth and regression of thalamic efferents in the song-control system of male zebra finches. J Comp Neurol. 1992; 326(3):442-450. [PubMed: 1469121]

19. Johnson F, Bottjer SW. Afferent influences on cell death and birth during development of a cortical nucleus necessary for learned vocal behavior in zebra finches. Development. 1994; 120:13-24. [PubMed: 21375056]

20. Johnson F, Sablan MM, Bottjer SW. Topographic organization of a forebrain pathway involved with vocal learning in zebra finches. J Comp Neurol. 1995; 358:260-278. [PubMed: 7560286]

21. Johnson F, Hohmann SE, DiStefano PS, Bottjer SW. Neurotrophins suppress apoptosis induced by deafferentation of an avian motor-cortical region. J Neurosci. 1997; 17:2101-2111. [PubMed: 9045737]

22. Kao MH, Brainard MS. Lesions of an avian basal ganglia circuit prevent context-dependent changes to song variability. J Neurophysiol. 2006; 96:1441-1455. [PubMed: 16723412]

23. Kittelberger JM, Mooney R. Lesions of an avian forebrain nucleus that disrupt song development alter synaptic connectivity and transmission in the vocal premotor pathway. J Neurosci. 1999; 19:9385-9398. [PubMed: 10531443]

24. Konishi M, Akutagawa E. Neuronal growth, atrophy and death in a sexually dimorphic song nucleus in the zebra finch brain. Nature. 1985; 315:145-147. [PubMed: 3990816]

25. Long MA, Fee MS. Using temperature to analyse temporal dynamics in the songbird motor pathway. Nature. 2008; 456:189-194. [PubMed: 19005546]

26. Long MA, Jin DZ, Fee MS. Support for a synaptic chain model of neuronal sequence generation. Nature. 2010; 468:394-399. [PubMed: 20972420]

27. Mooney R. Synaptic basis for developmental plasticity in a birdsong nucleus. J Neurosci. 1992; 12:2464-2477. [PubMed: 1351935] 
28. Naie K, Hahnloser RHR. Regulation of learned vocal behavior by an auditory motor cortical nucleus in juvenile zebra finches. J Neurophysiol. 2011; 106:291-300. [PubMed: 21525374]

29. Nordeen KW, Nordeen EJ. Sex and regional differences in the incorporation of neurons born during song learning in zebra finches. J Neurosci. 1988a; 8:2869-2874. [PubMed: 3411358]

30. Nordeen KW, Nordeen EJ. Projection neurons within a vocal motor pathway are born during song learning in zebra finches. Nature. 1988b; 334:149-151. [PubMed: 3386754]

31. Olveczky BP, Andalman AS, Fee MS. Vocal experimentation in the juvenile songbird requires a basal ganglia circuit. PLoS Biol. 2005; 3:e153. [PubMed: 15826219]

32. Roberts TF, Klein ME, Kubke MF, Wild JM, Mooney R. Telencephalic neurons monosynaptically link brainstem and forebrain premotor networks necessary for song. J Neurosci. 2008; 28:3479_ 3489. [PubMed: 18367614]

33. Roper A, Zann R. The Onset of Song Learning and Song Tutor Selection in Fledgling Zebra Finches. Ethology. 2006; 112:458-470.

34. Saar S, Mitra PP. A technique for characterizing the development of rhythms in bird song. PLoS One. 2008; 3:e146.

35. Scharff C, Nottebohm F. A comparative study of the behavioral deficits following lesions of various parts of the zebra finch song system: implications for vocal learning. J Neurosci. 1991; 11:2896-2913. [PubMed: 1880555]

36. Sober SJ, Brainard MS. Adult birdsong is actively maintained by error correction. Nat Neurosci. 2009; 12:927-931. [PubMed: 19525945]

37. Tchernichovski O, Nottebohm F, Ho CE, Pesaran B, Mitra PP. A procedure for an automated measurement of song similarity. Anim Behav. 2000; 59:1167-1176. [PubMed: 10877896]

38. Tchernichovski O, Mitra PP, Lints T, Nottebohm F. Dynamics of the Vocal Imitation Process: How a Zebra Finch Learns Its Song. Science. 2001; 291:2564-2569. [PubMed: 11283361]

39. Thompson JA, Basista MJ, Wu W, Bertram R, Johnson F. Dual pre-motor contribution to songbird syllable variation. J Neurosci. 2011; 31:322-330. [PubMed: 21209218]

40. Tritsch NX, Sabatini BL. Dopaminergic modulation of synaptic transmission in cortex and striatum. Neuron. 2012; 76:33-50. [PubMed: 23040805]

41. Vates GE, Nottebohm F. Feedback circuitry within a song learning pathway. Proc Natl Acad Sci U S A. 1995; 92:5139-5143. [PubMed: 7761463]

42. Wu W, Thompson JA, Bertram R, Johnson F. A statistical method for quantifying songbird phonology and syntax. J Neurosci Methods. 2008; 174:147-154. [PubMed: 18674560]

43. Zann R. Ontogeny of the zebra finch distance call: I. Effects of cross-fostering to Bengalese finches. Z Tierpsychol. 1985; 68:1-23.

Dev Neurobiol. Author manuscript; available in PMC 2015 June 01. 


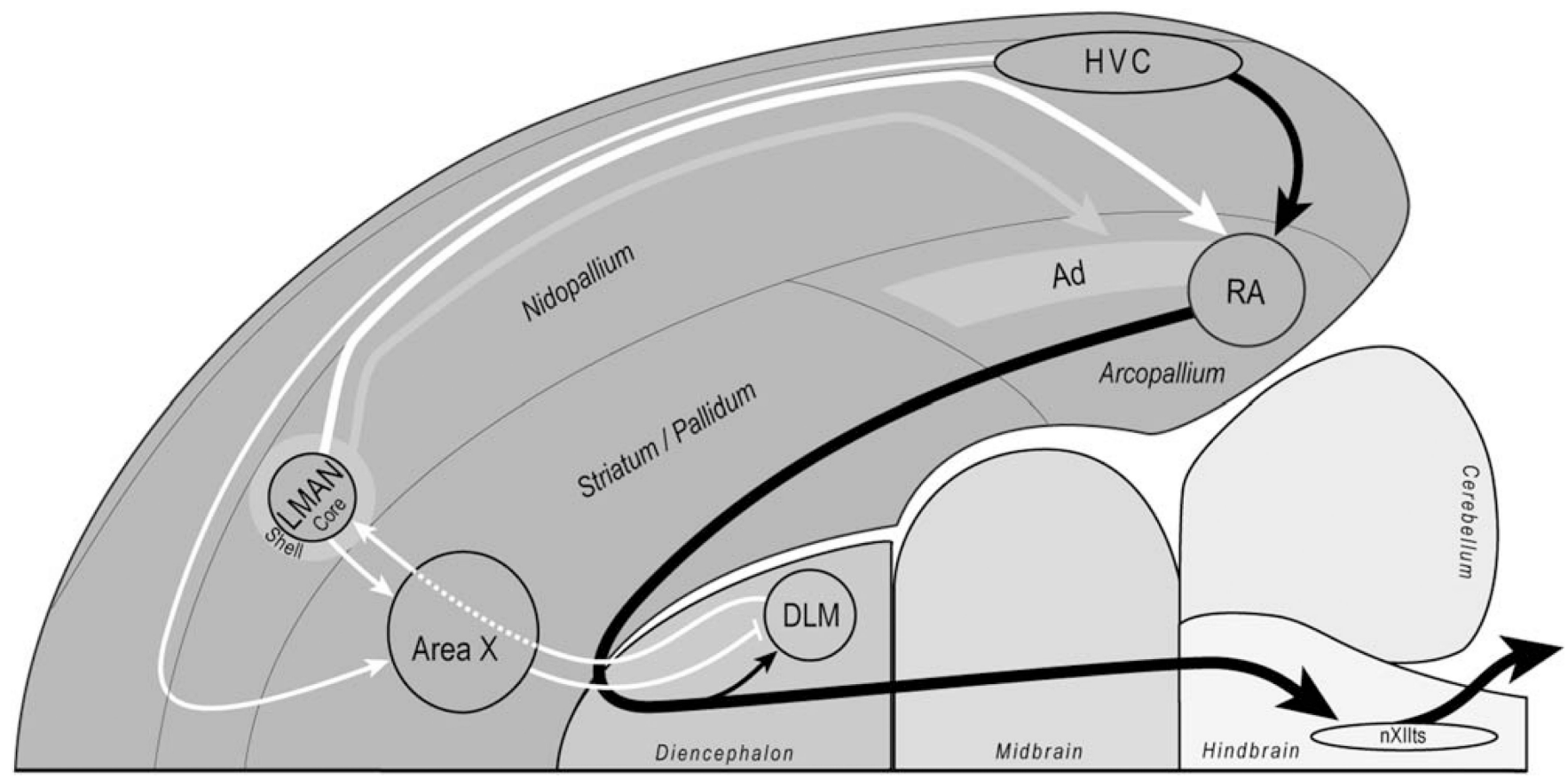

Figure 1. Sagittal schematic of the zebra finch song circuit

Antero-posterior axis is left to right and dorso-ventral axis is top to bottom. Schematic shows the anterior pathway (in white) and the posterior pathway (in black). Pallial regions of the avian brain (e.g., nidopallium, arcopallium) are analogous to mammalian cortex.

Abbreviations: Ad, dorsal arcopallium; DLM, dorsal lateral nucleus of the medial thalamus; HVC, proper name, located in the nidopallium; LMAN, lateral magnocellular nucleus of the anterior nidopallium; nXIIts, tracheosyringeal nerve; RA, robust nucleus of the anterior nidopallium. 
- LMAN intact

- - - LMAN ablated
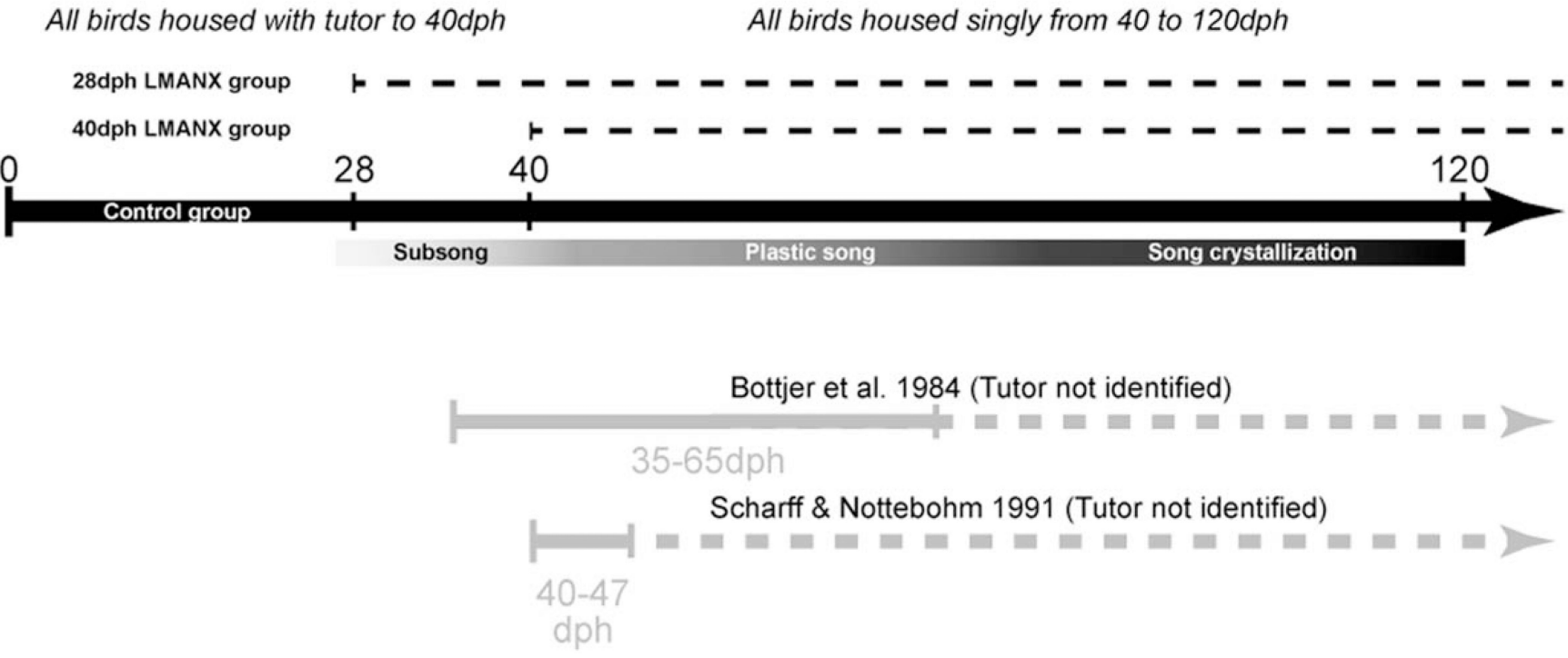

Figure 2. Timeline of the experiment

The central black line represents the lifespan of experimental and control subjects, ranging from 0 - to 120-dph (adulthood). Below the timeline is a bar showing the phases of sensorymotor development aligned to their normative onsets and offsets. All birds were housed with their tutor until 40dph and then housed singly to the end of the experiment. Experimental birds in our study received LMAN ablation at either 28- or 40-dph, prior to the production or differentiation of protosyllables, respectively. Timelines for other studies of juvenile LMAN ablation (Bottjer et al., 1984; Scharff \& Nottebohm, 1991) are included below for comparison. Solid lines indicate intact LMAN and dotted lines indicate ablated LMAN. 
A

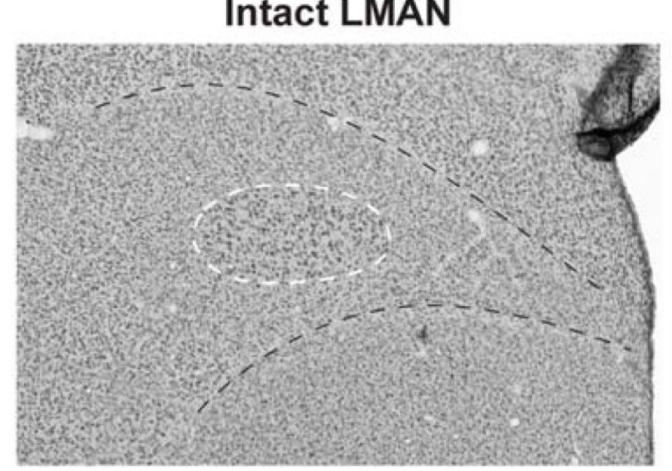

\section{Complete LMAN ablation}

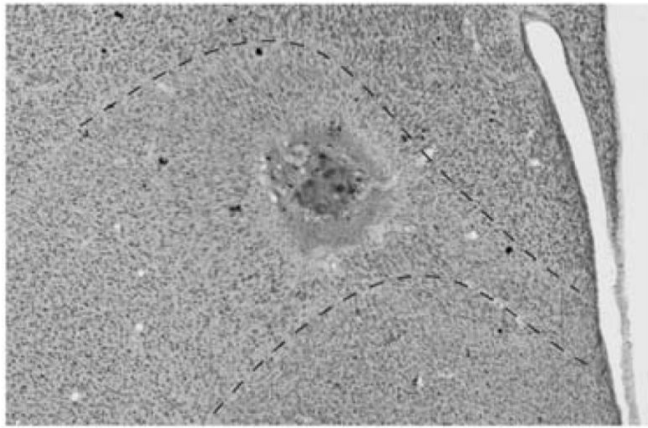

\section{B}

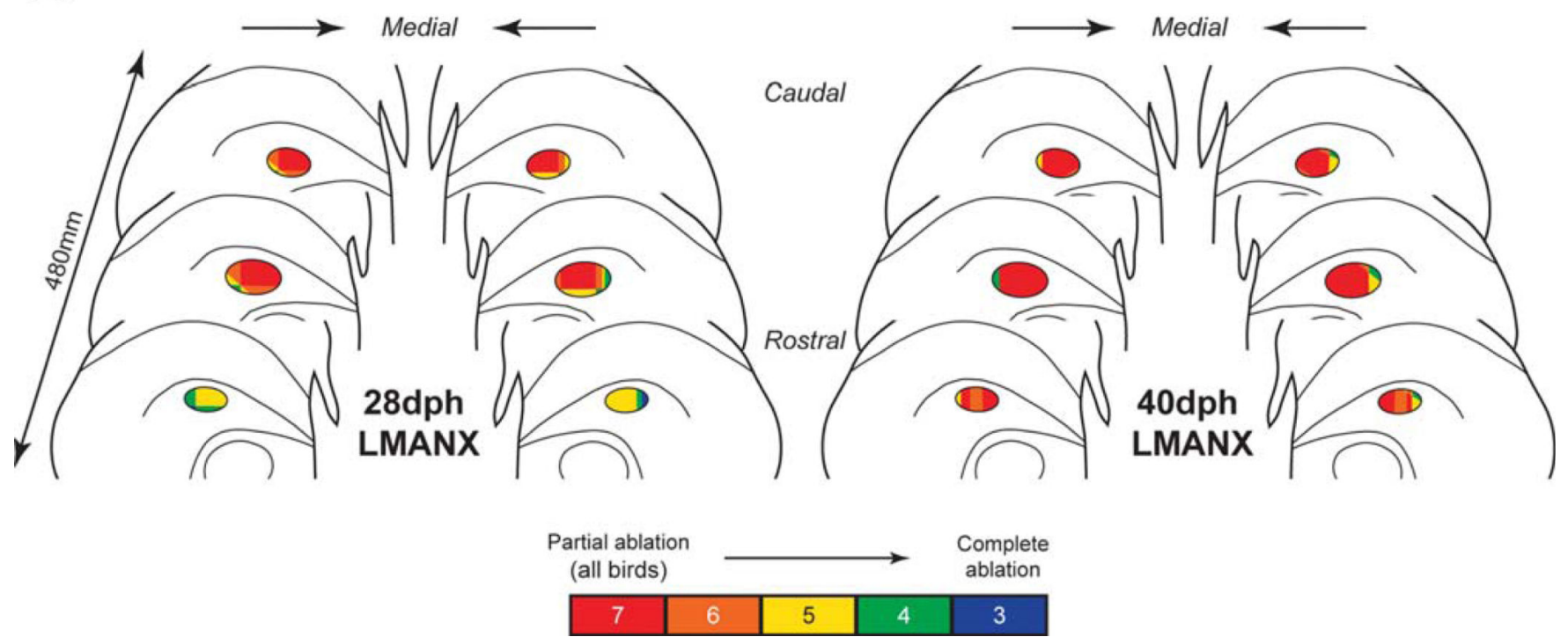

Figure 3. Overlay of bilateral coronal sections in both LMAN-ablation groups

(A) Representative images of intact LMAN (outlined in white, from a CTL bird) and a complete LMAN ablation are shown (from a 28dph bird). (B) Bilateral coronal sections for both 28- and 40- dph LMANX groups show overlapping LMAN ablation sites in all LMANX birds ( $\mathrm{N}=7$ in both 28 - and 40-dph groups). The position and amount of LMAN ablated for each bird were normalized relative to tracings of LMAN in CTL birds, and then combined to create the overlays. Color fills indicate regions of overlapping ablation across birds (e.g., all seven birds in each group received ablation in red-shaded regions of LMAN). Three birds in each group received complete LMAN ablation. The pattern of overlap indicates that LMAN ablation occurred most consistently in caudal and central LMAN. 


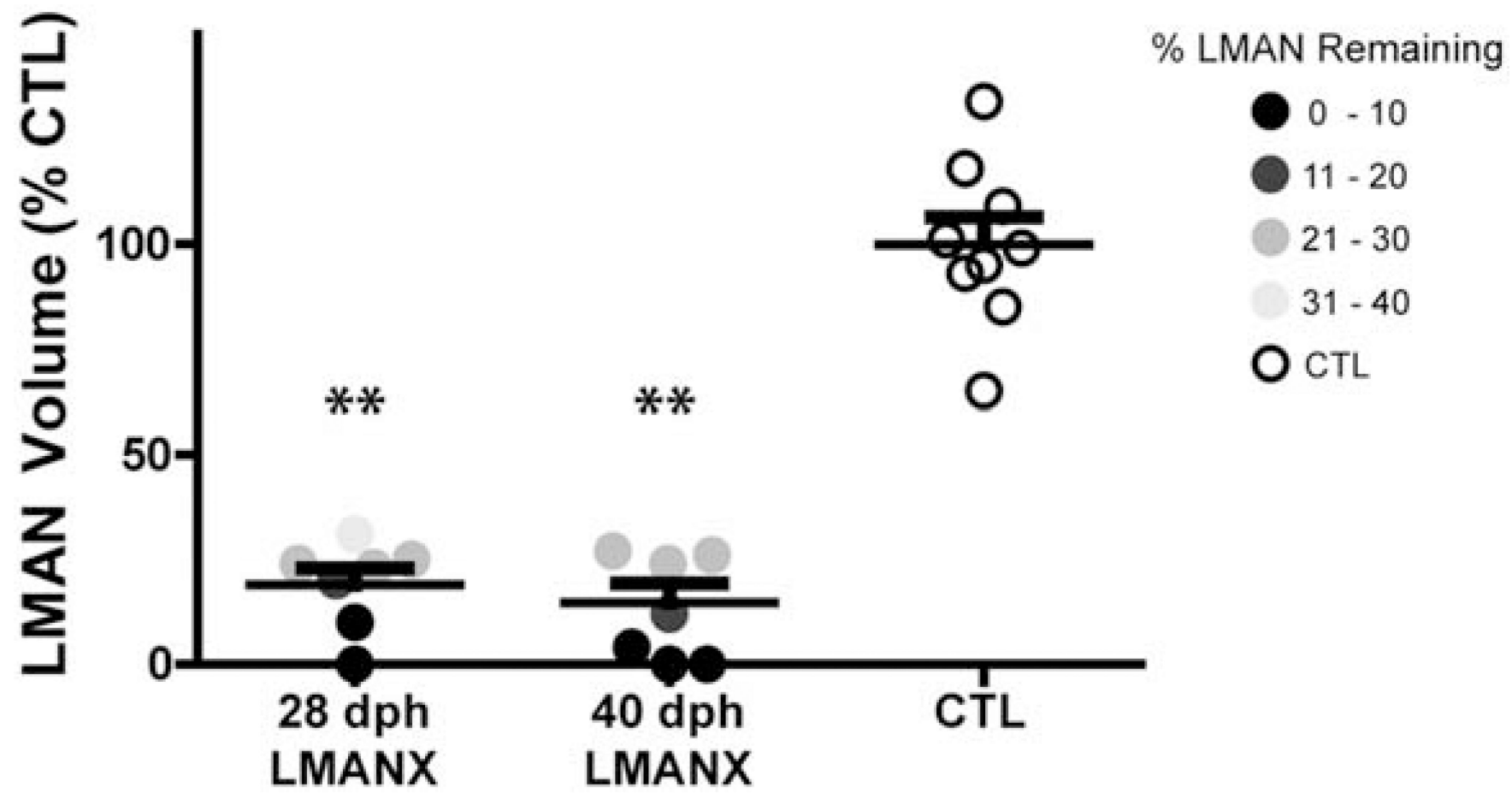

Figure 4. Confirmation of LMAN ablation

All birds in the 28- and 40-dph LMANX groups had significantly smaller LMAN volume compared to the CTL group (all volumes calculated as a percentage of the mean LMAN volume in the CTL group). Long and short bars indicate mean and S.E.M., respectively. Asterisks represent significance values of $\mathrm{p} \leq 0.01(* *)$. 

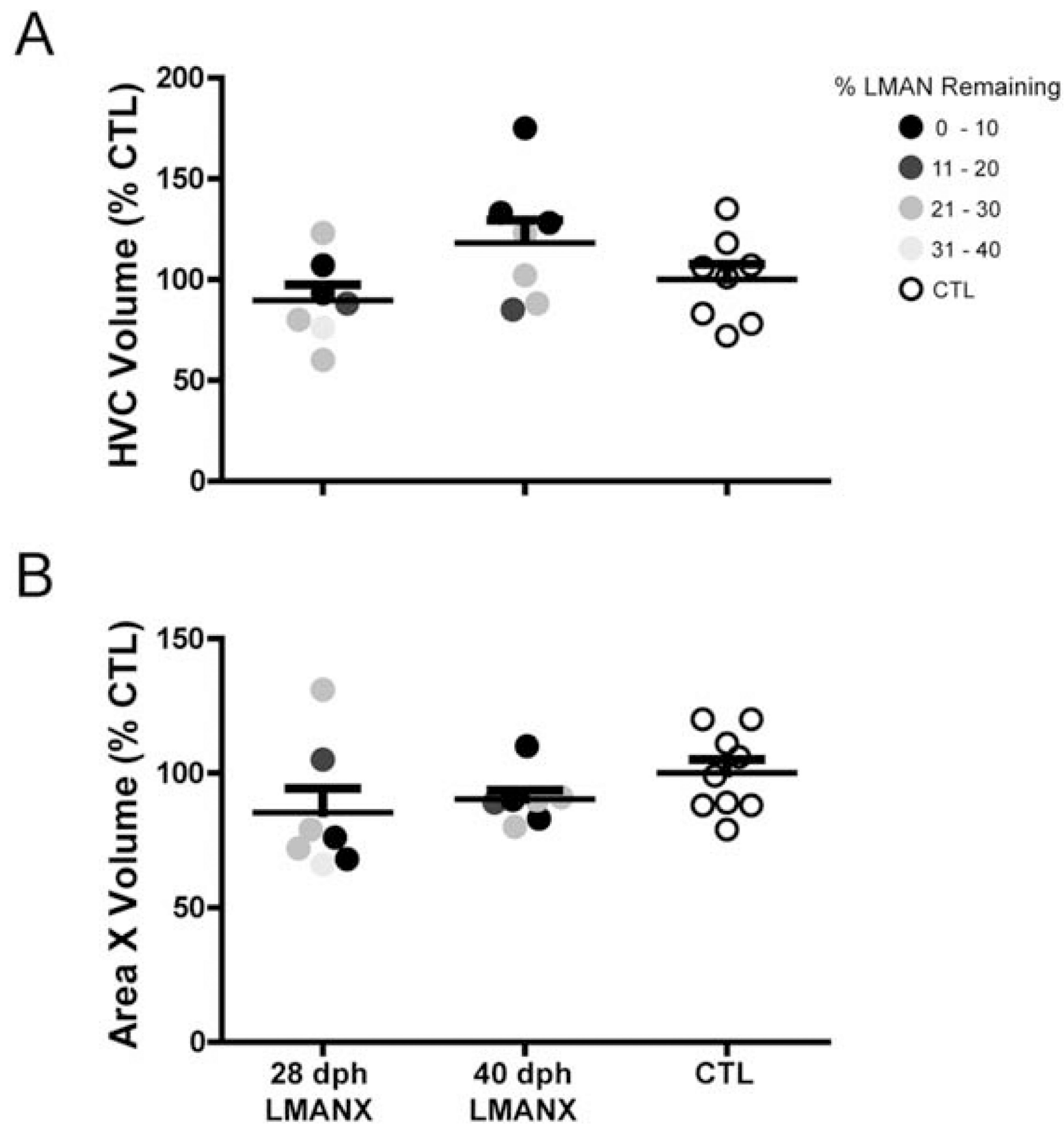

Figure 5. Juvenile LMAN ablation does not influence the development of HVC and Area X morphology

Juvenile LMAN ablation did not significantly influence the adult volume of (A) HVC and (B) Area X in either the 28- or 40-dph LMANX groups (volumes calculated as a percentage of the mean volume in the CTL group). Long and short bars indicate mean and S.E.M., respectively. 


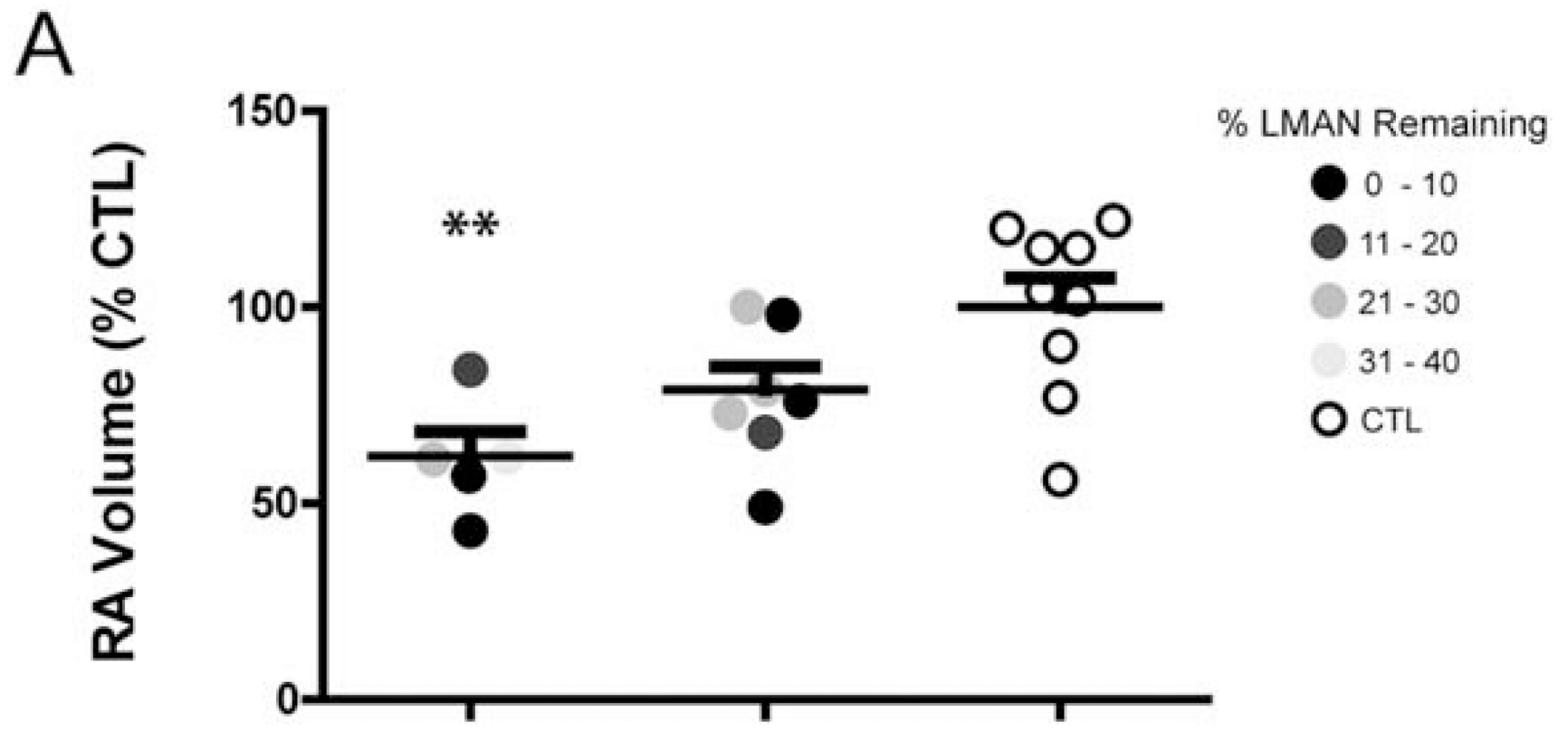

B

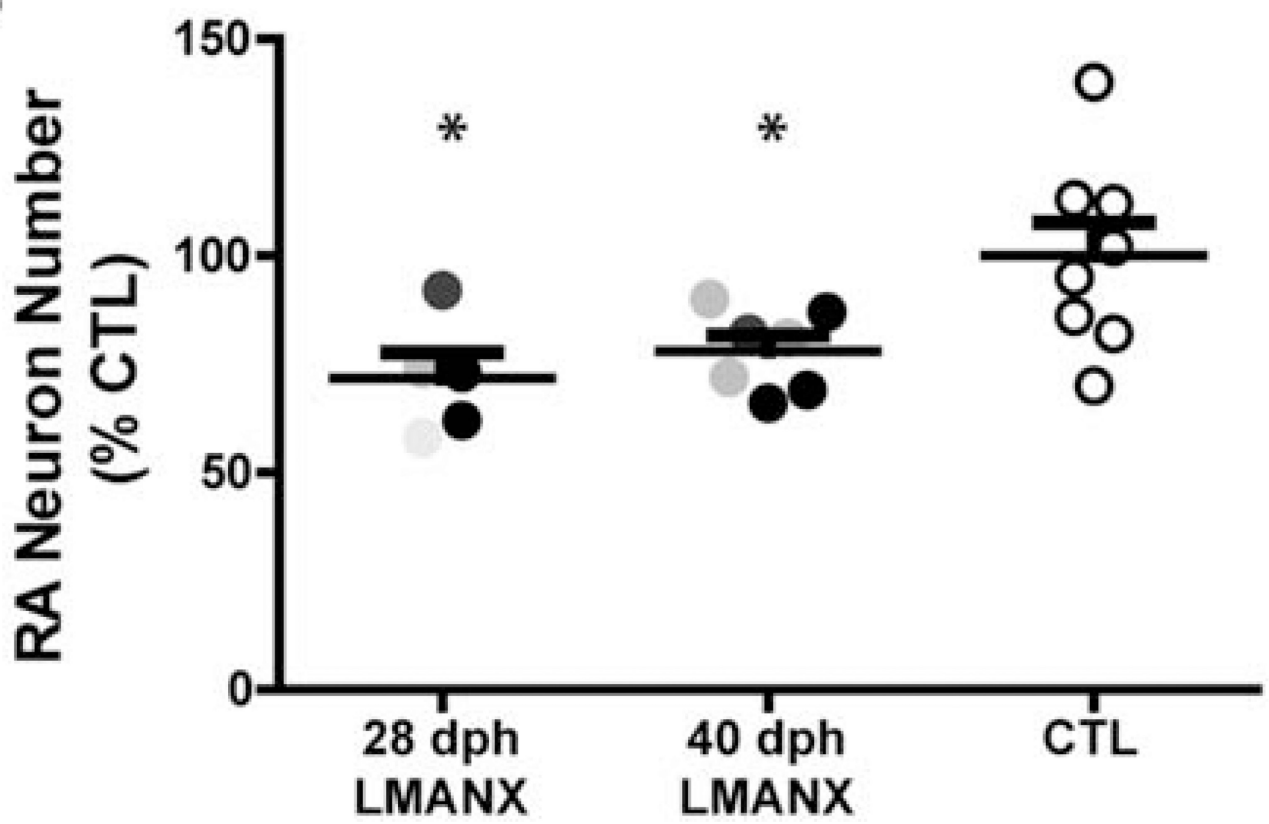

Figure 6. Juvenile LMAN ablation alters the morphological development of RA

(A) Compared to birds in the CTL group, birds in the 28dph LMANX group had a significantly reduced RA volume (calculated as a percentage of the mean RA volume in the CTL group). RA volumes from birds in the 40-dph LMANX group were not significantly different from the CTL group. (B) Both 28- and 40-dph LMANX groups had significantly fewer RA neurons than the CTL group (calculated as a percentage of the mean RA neuron number in the CTL group). Long and short bars indicate mean and S.E.M., respectively. Asterisks represent significance values of $\mathrm{p} \leq 0.05(*)$ or $\mathrm{p} \leq 0.01(* *)$. 
Tutor 309
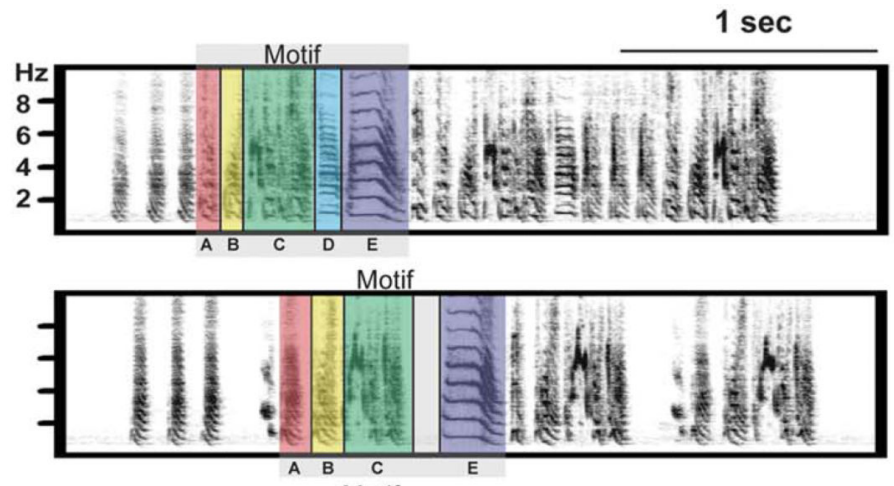

Son 309F

Control

Motif

Son $309 Q$

Control

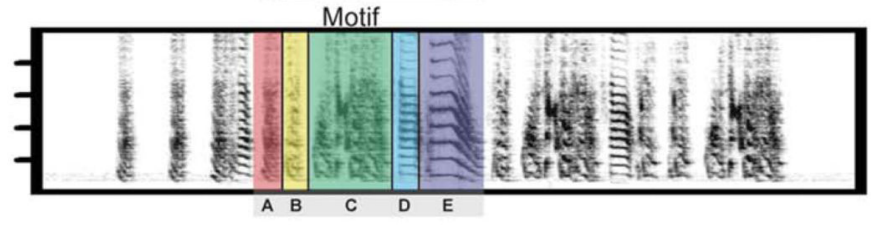

$24 \%$ LMAN remaining

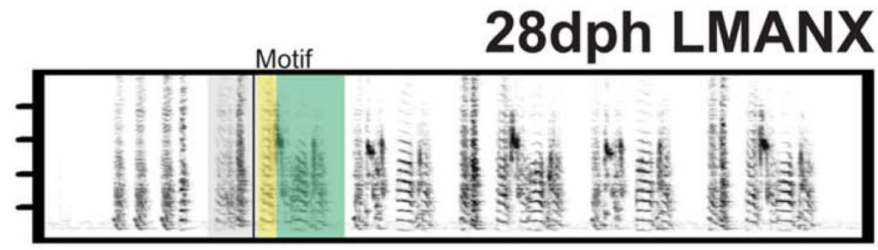

Son 3090

0\% LMAN remaining

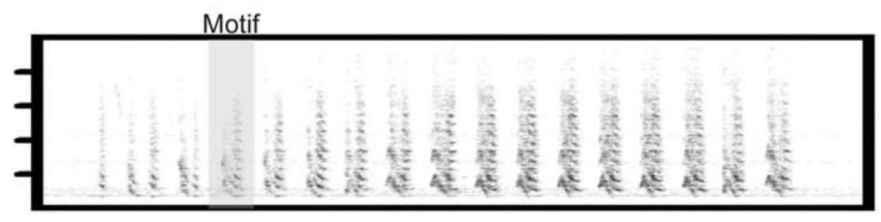

Son 309Y

$27 \%$ LMAN remaining
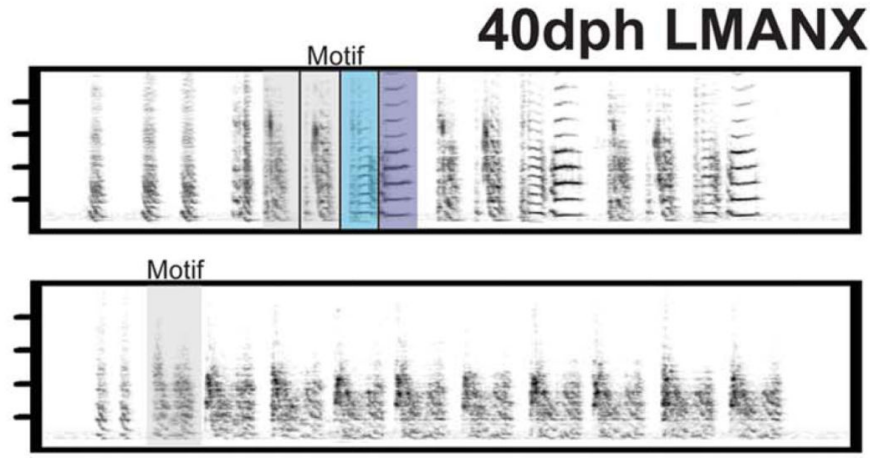

Figure 7. LMAN ablation at 28- or 40-dph impairs accuracy, duration and amplitude of tutor song imitation

Below the tutor's spectrogram are letters, each corresponding to a different syllable within the tutor's song motif. Tutor syllables copied by CTL sons are indicated below their respective spectrograms. Transparent colors indicate syllables that match specific tutor syllables. Transparent colors without letters in spectrograms from birds with partial LMAN ablation (309V and 309Y) indicate the closest resembling tutor syllables. Birds with juvenile LMAN ablation at either 28- or 40-dph have shortened motifs that contained fewer syllables, produced at reduced amplitude (indicated by lighter gray-scaling). Recordings of tutor songs 
and of the final adult songs of CTL and LMANX birds were made in the same acousticisolation chamber with the cage, microphone and microphone gain set at fixed positions; this procedure enabled direct comparison of song amplitude between birds. 


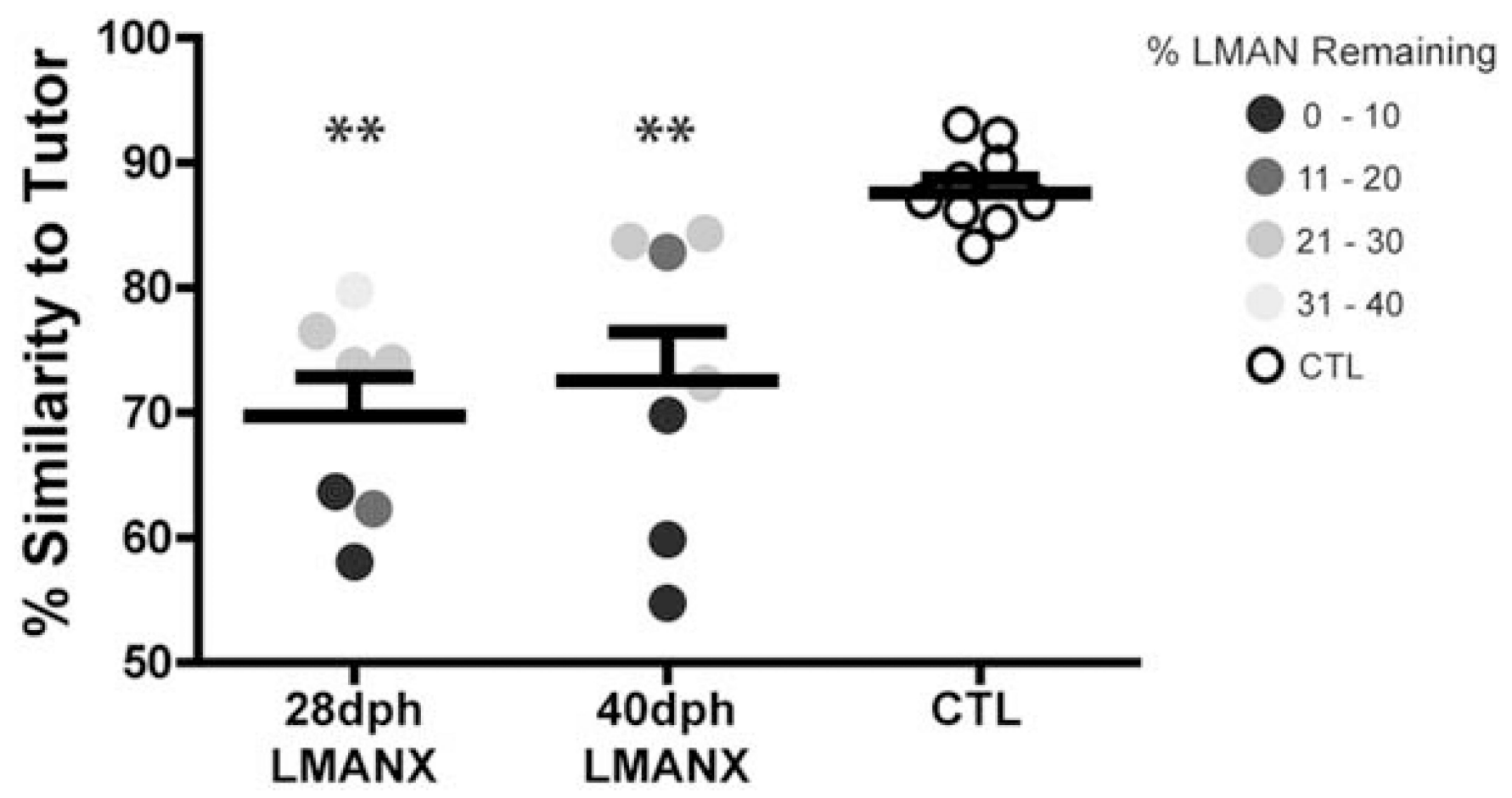

Figure 8. LMAN ablation at 28- or 40-dph impairs imitation of the spectral structure of tutor song

When compared to the CTL group, songs in the 28- and 40-dph LMANX groups were significantly less similar to their tutor's song, indicating that vocal imitation varied as a function of LMAN ablation. Within both 28- and 40-dph LMANX groups, similarity to tutor shows a positive correlation with the amount of LMAN remaining (lighter circles indicative of more LMAN remaining show greater similarity to the tutor's song). Long and short bars indicate mean and S.E.M., respectively. Asterisks represent significance value of $\mathrm{p} \leq 0.01$ $(* *)$. In addition, percent similarity to the tutor song is correlated with the amount of LMAN remaining following ablation when data from both 28 - and 40-dph groups are combined $\left(\mathrm{R}^{2}\right.$ $=0.45, \mathrm{p}<0.01)$. 


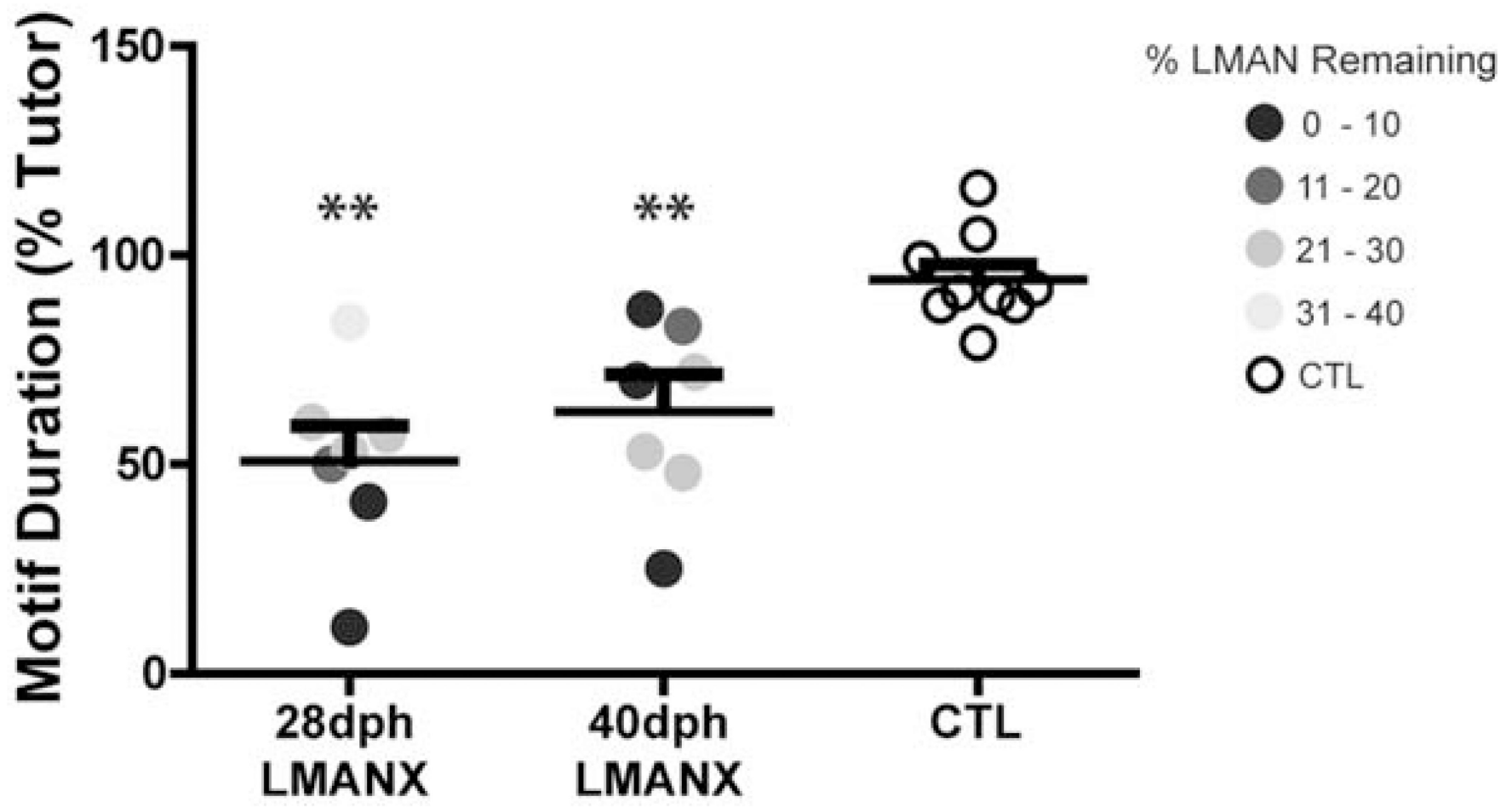

Figure 9. LMAN ablation at 28- or 40-dph produces a similar attenuation of motif duration Whereas CTL birds successfully imitated the duration of their tutor's motif, LMAN ablation at 28- or 40-dph significantly attenuated imitation of the duration of the tutor's motif. Long and short bars indicate mean and S.E.M., respectively. Asterisks represent significance value of $\mathrm{p} \leq 0.01(* *)$. 

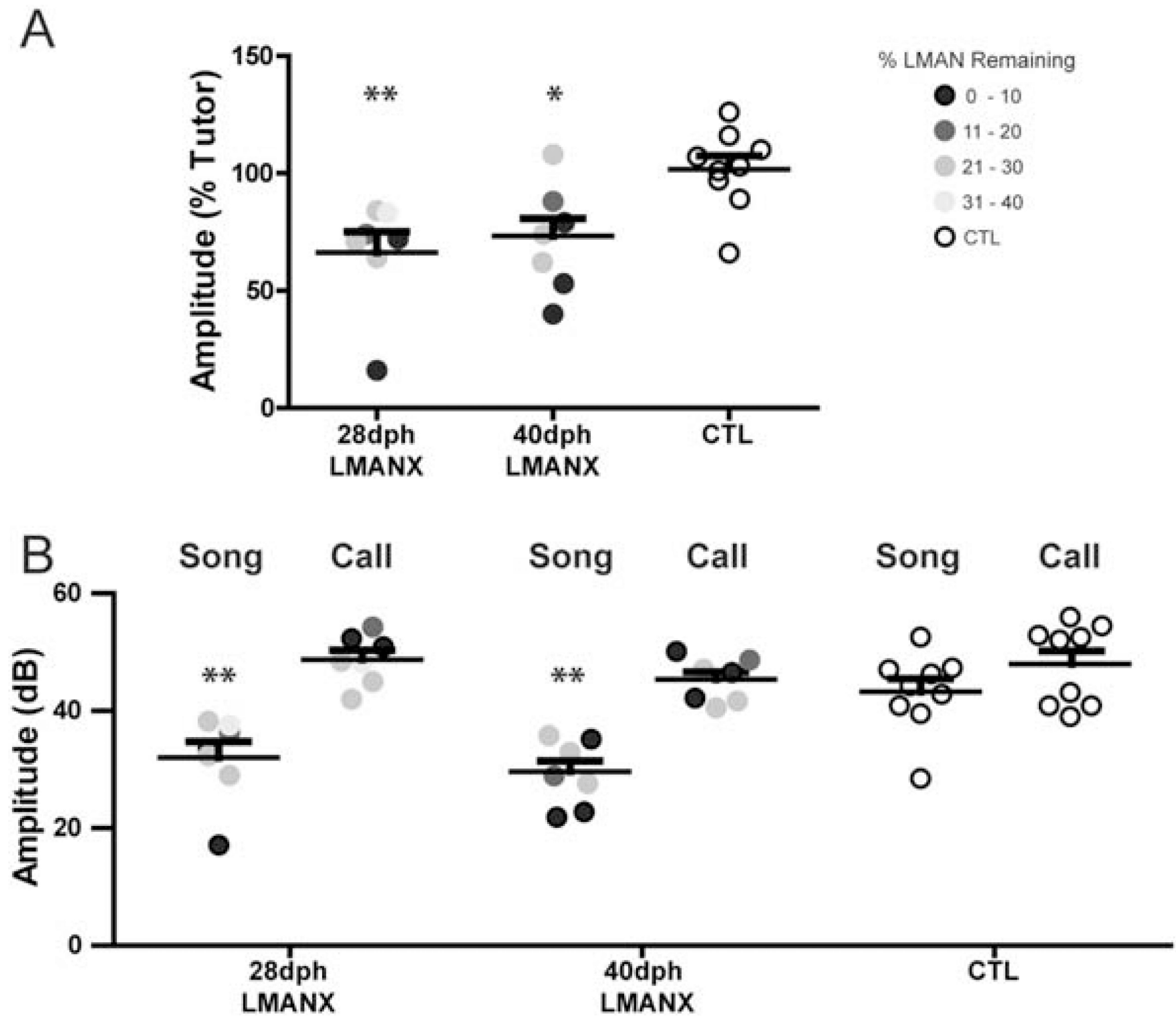

Figure 10. LMAN ablation at 28- or 40-dph produces a similar attenuation of motif amplitude (A) Whereas CTL birds successfully imitated the amplitude of their tutor's motif, LMAN ablation at 28- and 40-dph significantly attenuated imitation of the amplitude of the tutor's motif. (B) Amplitude (in decibels, dB) measures of the motif and distance calls of all birds show that the amplitude of motifs - but not distance calls - varied as a function of LMAN ablation. Thus, the effects of LMAN ablation were specific to song and did not produce general vocal-motor impairment. Long and short bars indicate mean and S.E.M., respectively. Asterisks represent significance values of $\mathrm{p} \leq 0.05(*)$ or $\mathrm{p} \leq 0.01(* *)$. 


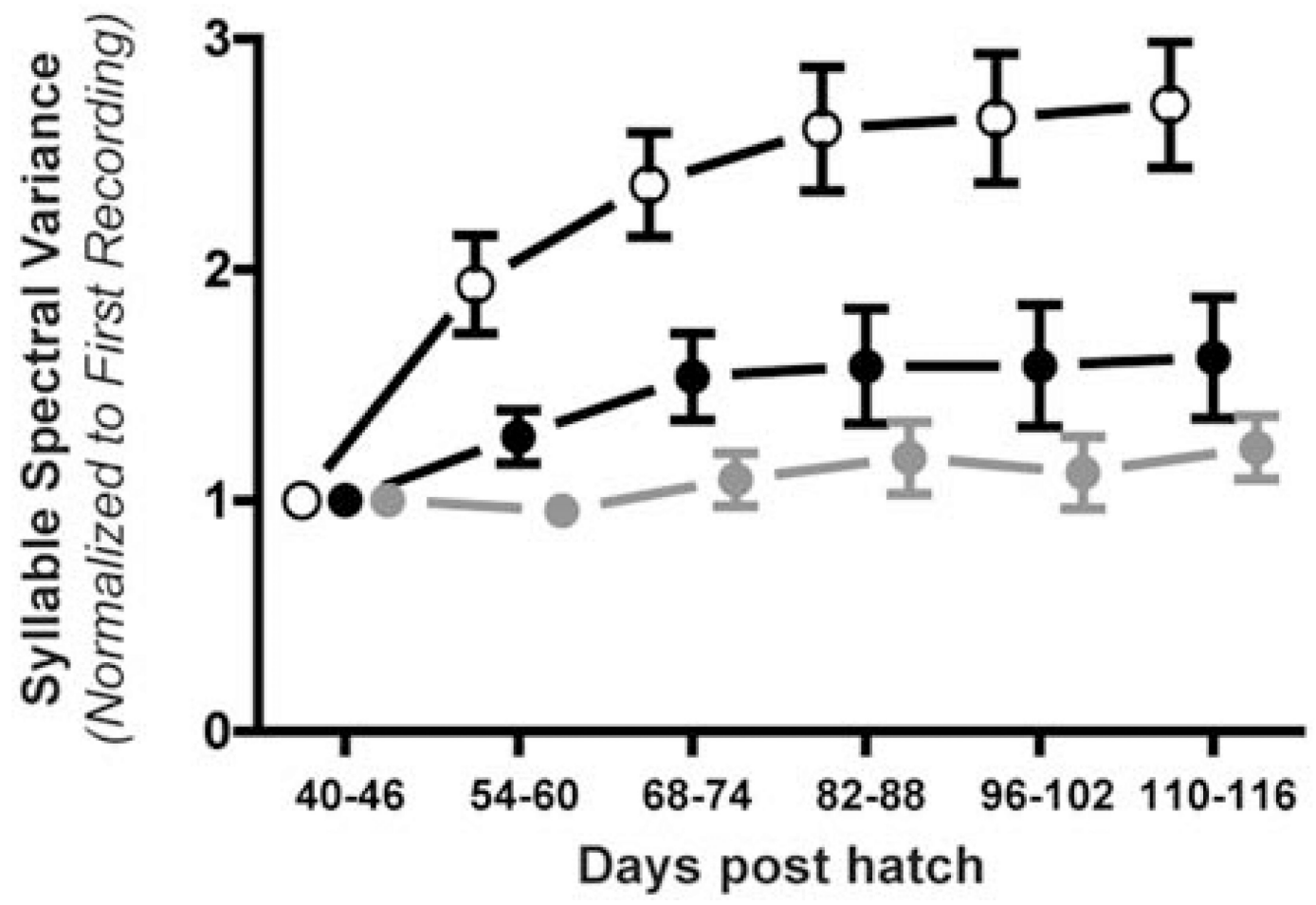

O CTL

Figure 11. LMAN ablation at 28- or 40-dph produces a similar attenuation of the learningrelated increase in syllable spectral complexity

Data points within each group represent the syllable spectral variation (consisting of normalized and averaged pitch, pitch goodness, and entropy variances) for that week for all birds. The spectral development of both 28- and 40-dph LMANX groups show an attenuation of the learning-related increase in syllable spectral complexity observed in CTL birds. Data points are means and error bars indicate S.E.M. 

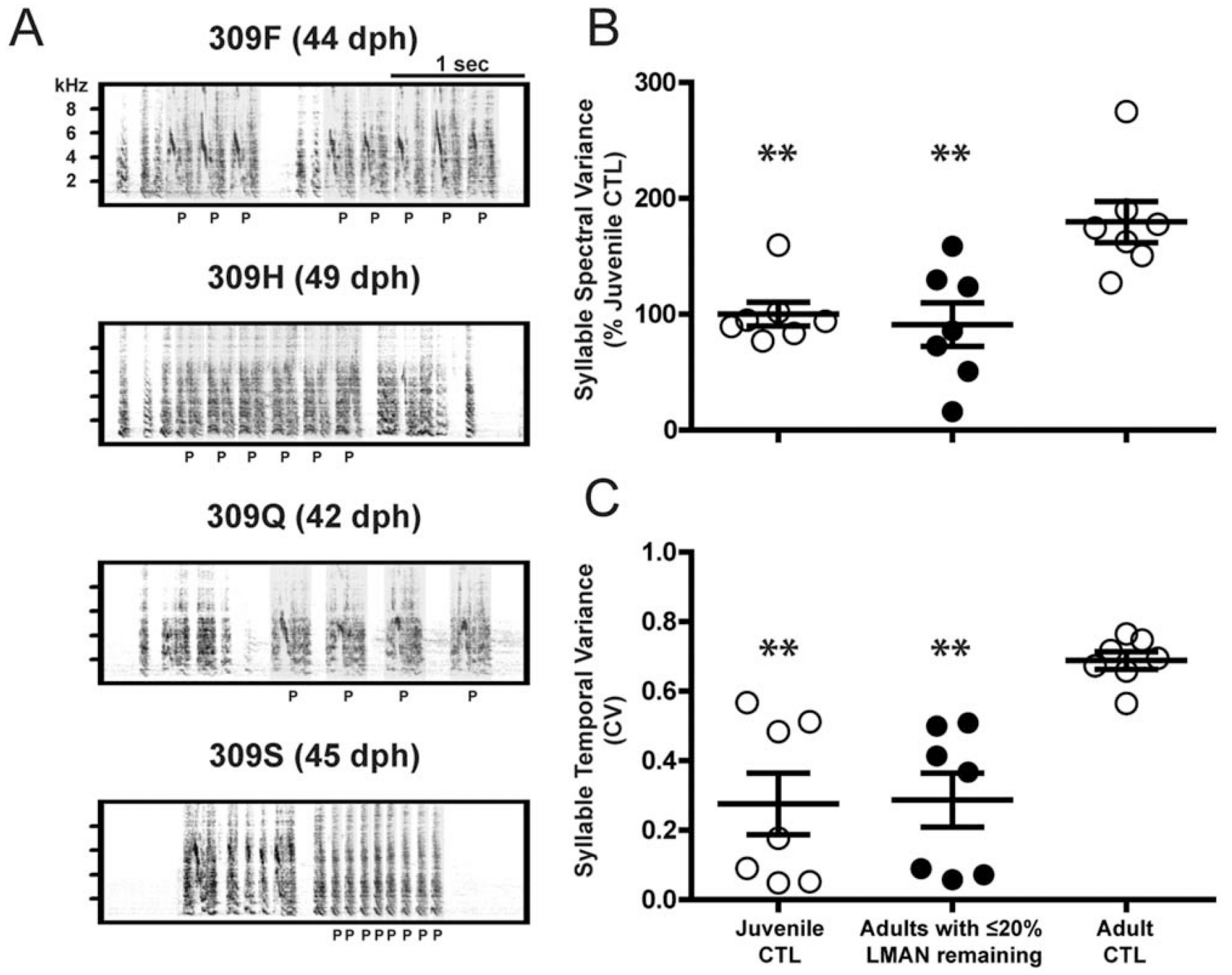

Figure 12. Song syllables produced by adult LMANX birds resemble protosyllables produced by juvenile CTL birds

(A) The spectral and temporal structure of juvenile CTL protosyllables resembles that of adult birds with complete LMAN ablation (compare to 3090 and 309W in Fig. 7). Each spectrogram shows examples of protosyllables produced by four different juvenile CTL birds during early plastic song (42-49 dph). The letter 'P' below each spectrogram corresponds to each bird's respective protosyllable. (B) Protosyllables produced by juvenile CTL birds and the adult syllables of birds with $\_20 \%$ LMAN lack the spectral variety of the syllables of adult CTL birds, but are not different from each other. (C) Protosyllables produced by juvenile CTL birds and the adult syllables of birds with $ک 20 \%$ LMAN remaining lack the temporal variety (expressed as $\mathrm{CV}$, the coefficient of variation) of the syllables of adult CTL birds, but are not different from each other. Long and short bars indicate mean and S.E.M., respectively. Asterisks represent significance values of $\mathrm{p} \leq 0.01$ (**). 